\title{
On the Coherent Radiation of an Electron Bunch Moving in an Arc of a Circle
}

\author{
E.L. Saldin ${ }^{\text {a }}$, E.A. Schneidmiller ${ }^{\text {a }}$, M.V. Yurkov ${ }^{b}$ \\ a Automatic Systems Corporation, 443050 Samara, Russia \\ b Joint Institute for Nuclear Research, Dubna, 141980 Moscow Region, Russia
}

\begin{abstract}
Existing theories of coherent synchrotron radiation (CSR) are related to the motion of an electron bunch on a circular orbit and do not describe the case of finite magnet length. In this paper we present the CSR theory for a bunch of any length moving in an arc of a finite angle. The radiative interaction of the electrons in the bunch is analyzed for a line charge distribution using ultrarelativistic approximation. It is shown in particular that this interaction is important not only inside the magnet but also on the straight part of the trajectory after the magnet.

Detailed analytical study of the CSR effects in the electron bunch with a stepped distribution of the charge density has been performed. The simple analytical technique of the radiative force calculation has been developed. The analytical solutions in the form of elementary functions are obtained for the radiative interaction force, for the energy loss distribution along the bunch and for the total energy loss of the bunch. The latter result is confirmed with calculation of the energy of coherent radiation in far zone. The criterium for the applicability region of the preceding theories to the case of a finite magnet length is obtained.
\end{abstract}




\section{Introduction}

Fifty years ago the development of the theory of synchrotron radiation has been stimulated significantly due to rapid progress in the construction of electron synchrotrons, and nowadays the development of the theory of coherent synchrotron radiation (CSR) is stimulated by the need to construct future generation linear colliders [1]. The bunch length in the main accelerator of linear collider should be about $l_{b} \sim 0.1-1 \mathrm{~mm}$ at the value of the bunch charge of few $\mathrm{nC}$. These values exceed by an order of magnitude those used previously. The CSR effects are also important for designers of X-ray FELs [2-4]. These projects require even shorter bunches, down to $0.025 \mathrm{~mm}$. Such intensive and short bunches are obtained by multi-stage compression in special magnetic chicanes (bunch-compressors). Analytical estimations and numerical simulations show that the effects of coherent synchrotron radiation become to be significant in such systems and even could prevent the achievement of the required beam parameters leading to the growth of the energy spread and emittance dilution $[5,6]$. The problem of coherent synchrotron radiation of a short bunch in an undulator should be studied, too. Nowadays the development of the linear collider projects entered into the stage of construction of test facilities for testing main technical solutions [7] and the project of $6 \mathrm{~nm}$ VUV SASE FEL is now under construction at DESY [4]. The success of construction of theses facilities depends significantly on correct understanding of CSR effects influence on the beam dynamics.

The first works on the theory of coherent synchrotron have been performed fifty years ago [8-10]. In these papers the main emphasis was put on the calculations in far zone of CSR produced by a bunch of relativistic electrons moving on a circular orbit. Another part of the problem, namely that of the radiative interaction of electrons within a bunch has been studied for the first time in refs. $[11,12]$ and later in refs. $[5,13]$ where the energy losses along the bunch has been calculated. It was shown in these papers that the rate of the integral energy losses in the bunch coincides with the CSR power in far zone $[8-10]$.

It should be noticed that all the results obtained in refs. [5,8-13] are valid only for the model situation of circular motion of a bunch. In practice accelerator systems are composed of bending magnets separated by straight sections and the trajectory of the electron bunch is not a circular one, but consists of a number of arcs joined by straight sections. So, a reasonable question arises about the possibility to describe this case with the results obtained for the model situation of a circular motion. A rough estimation for the applicability region of the latter results for the case of a bending magnet are as follows:

$$
R / \gamma^{3} \ll l_{b} \ll R \phi_{m}^{3} / 24
$$


where $l_{b}$ is the length of the electron bunch, $R$ is the bending radius of the magnet, $\phi_{m}$ is the bending angle of the particle in the magnet $\left(\phi_{m} \ll 1\right), \gamma$ is relativistic factor $(\gamma \gg 1)$ and $s_{m}=R \phi_{m}^{3} / 24$ is the slippage length equal to the path difference of the curved trajectory inside the magnet and the straight line between entrance and exit points of the magnet. So, we can conclude that this applicability region is a narrow one and the results of refs. $[5,8-13]$ could be used carefully in each practical situation. For instance, it could be shown that some accelerator systems of linear colliders and X-ray FELs fall out of applicability region given by eq (1).

In the present paper we extend the study of CSR effects for the case of the electron bunch of an arbitrary length $l_{b}$ passing isolated bending magnet of small bending angle $\phi_{m}$. We consider the model of ultrarelativistic electron bunch with a linear distribution of the charge (zero transverse dimensions). We perform detailed study of the CSR effects in the electron bunch with a stepped distribution of the charge density. To calculate the radiative interaction force we have developed the analytical technique of complementary integral which can be used also in more complicated cases (a sequence of magnets, an undulator etc.). Using this technique we have obtained analytical expressions in the form of elementary functions for the longitudinal force, for the distribution of the energy losses along the bunch and for the integral energy losses of the bunch. The results for the integral energy losses of the bunch are in complete agreement with the results of the integration of spectral density of the radiation energy in far zone. The results obtained in this paper allow one to impose more correct limitations (with respect to the estimation given by eq. (1)) on the applicability region of the results obtained in the framework of the model of a circle motion of the electron bunch [5,8-13].

The simple physical model considered in this paper, does not allow one to take into account a number of physical effects influencing on the process of coherent radiation. These effects refer to finite transverse dimensions of the bunch, to the shielding of radiation due to the presence of vacuum chamber, to the influence of adjacent bending magnets, etc. Nevertheless, giving simple analytical relations, such a model allows one to get a deeper insight into the physics of CSR. It provides also a firm base for a quick estimation for an upper limit of power of CSR effects. If such an estimation will indicate on the strong influence of CSR effects on the beam dynamics, numerical simulations codes, taking into account the realistic situation should be used to obtain more correct result. Analytical results could serve in this case as a primary standard for testing these numerical codes.

The paper is organized as follows. The radiative interaction of two electrons executing a circular motion is analyzed in section 2 and physically transparent method of radiative force calculation is presented. In section 3 this method is used for calculations of the radiative interaction of electrons in a bunch 
moving on a circle. In section 4 we study the radiative interaction of two electrons passing the bending magnet of finite length. In section 5 and 6 we study in detail the CSR effects in the electron bunch with the stepped profile. The results obtained in the paper are discussed in section 7 . It is illustrated how the technique for the calculation of radiation forces can be extended for the case of an arbitrary density profile of the bunch.

\section{Radiative interaction of two electrons moving on a circle}

We begin our study with the system of two particles moving on a circle of radius $R$. The electric field of a back electron produced at a source point $P^{\prime}$ at time $t^{\prime}$ reaches a front electron at time $t$ in the point $P$. This is described by the Lienard-Wiechert formula [15]:

$$
\vec{E}(P)=\frac{e}{\gamma^{2}} \frac{\left(\vec{n}-\vec{\beta}^{\prime}\right)}{L^{2}\left(1-\vec{n} \circ \vec{\beta}^{\prime}\right)^{3}}+\frac{e}{c^{2}} \frac{\vec{n} \times\left[\left(\vec{n}-\vec{\beta}^{\prime}\right) \times \vec{g}^{2}\right]}{L\left(1-\vec{n} \circ \vec{\beta}^{\prime}\right)^{3}}
$$

where

$$
1-\vec{n} \circ \vec{\beta}^{\prime}=1-\beta \cos \frac{\left(\phi-\phi^{\prime}\right)}{2},
$$

$\left(\phi-\phi^{\prime}\right), L$ and $\vec{n}$ are, respectively, the angle, the distance, and the unit vector directed from point $P^{\prime}$ to point $P, \vec{\beta}^{\prime}=\vec{v}^{\prime} / c, \vec{v}^{\prime}$ is the velocity at point $P^{\prime}$. The first term in the right-hand side of eq. (2) refers usually as velocity (or Coulomb) field and the second one, proportional to the acceleration $g^{\prime}=v^{2} / R$, refers as acceleration field (or radiation field).

Taking into account eq. (2) we can write the expression for the rate of the energy change of the front electron:

$$
\begin{aligned}
e \vec{v}(P) \circ \vec{E}(P)= & \frac{e^{2} c}{\gamma^{2}} \frac{\beta \cos \frac{\left(\phi-\phi^{\prime}\right)}{2}-\beta^{2} \cos \left(\phi-\phi^{\prime}\right)}{\left(2 R \sin \frac{\left(\phi-\phi^{\prime}\right)}{2}\right)^{2}\left(1-\beta \cos \frac{\left(\phi-\phi^{\prime}\right)}{2}\right)^{3}}+ \\
& \frac{e^{2}}{c^{2}} \frac{\left(\beta \cos \frac{\left(\phi-\phi^{\prime}\right)}{2}-\beta^{2} \cos \left(\phi-\phi^{\prime}\right)\right) g^{\prime} \sin \frac{\left(\phi-\phi^{\prime}\right)}{2}}{2 R \sin \frac{\left(\phi-\phi^{\prime}\right)}{2}\left(1-\beta \cos \frac{\left(\phi-\phi^{\prime}\right)}{2}\right)^{3}}- \\
& \frac{e^{2}}{c^{2}} \frac{g^{\prime} \sin \left(\phi-\phi^{\prime}\right)}{2 R \sin \frac{\left(\phi-\phi^{\prime}\right)}{2}\left(1-\beta \cos \frac{\left(\phi-\phi^{\prime}\right)}{2}\right)^{2}} .
\end{aligned}
$$


In this paper we use ultrarelativistic approximation for the electron motion and assume the bending angle of the particle in the magnet to be small. Under these conditions eq. (3) is reduced to:

$$
e \vec{v}(P) \circ \vec{E}(P)=\frac{e^{2} c}{R^{2}}\left[\frac{1-\beta+\frac{3 u^{2}}{8}}{\gamma^{2} u^{2}\left(1-\beta+\frac{u^{2}}{8}\right)^{3}}+\frac{\frac{u^{2}}{8}-(1-\beta)}{2\left(1-\beta+\frac{u^{2}}{8}\right)^{3}}\right] .
$$

where the retarded angle $u=\left(\phi-\phi^{\prime}\right)$ and the distance $\left(s-s^{\prime}\right)$ between electrons are connected by the relation:

$$
\left(s-s^{\prime}\right)=(1-\beta) R u+\frac{R u^{3}}{24} \text {. }
$$

The first term in the right-hand side of this relation appears due to a difference between the velocity of the electron and the velocity of light and the second one is equal to the difference in the length between the arc and the cord $P^{\prime} P$.

Analysis of expression (4) shows that the first term in square brackets contains singularity at $u=0$ (i.e. at $s=s^{\prime}$ ). In this paper we study pure radiative effects and remove this singularity using the following trick. We consider the system of two electrons moving along a straight line. In this case the rate of the energy change of the front electron is

$$
\frac{d \mathcal{E}}{d(c t)}=\frac{\epsilon^{2}}{\gamma^{2}\left(s-s^{\prime}\right)^{2}}
$$

and this expression contains singularity, too. We define the radiative force as the difference between eq. (4) and eq. (6):

$$
\begin{aligned}
\left(\frac{d \mathcal{E}}{d(c t)}\right)_{C S R}= & \frac{e^{2}}{R^{2}}\left\{\frac{\frac{u^{2}}{8}-(1-\beta)}{2\left(\frac{u^{2}}{8}+1-\beta\right)^{3}}+\right. \\
& \left.\frac{1}{\gamma^{2} u^{2}}\left[\frac{\frac{3 u^{2}}{8}+1-\beta}{\left(\frac{u^{2}}{8}+1-\beta\right)^{3}}-\frac{1}{\left(\frac{u^{2}}{24}+1-\beta\right)^{2}}\right]\right\},
\end{aligned}
$$

which does not contain singularity at $u=0$ and tends to zero in the limit of linear motion at $R \Rightarrow \infty, u \Rightarrow 0$ and $R u \Rightarrow\left(s-s^{\prime}\right) /(1-\beta)=$ const. In the following we will refer to the term in eq. (7) proportional to $1 / \gamma^{2} u^{2}$ as to "renormalized Coulomb term". 
The force acting on the back electron from the side of the front one is only of Coulomb nature and is described with the following expression independently of the trajectory type (a straight line or a circle):

$$
\frac{d \mathcal{E}^{\prime}}{d(c t)}=-\frac{e^{2}}{\gamma^{2}\left(s-s^{\prime}\right)^{2}} \text {. }
$$

So, we have excluded from consideration nondissipative interaction forces. The radiative force acting on the front electron is described with the eq. ( 7 ) and there is no radiative force acting on the back one.

Now we study the dependence of the radiative force $(7)$ on the distance between the electrons. We start with the asymptote of a small distance $\left(s-s^{\prime}\right) \ll$ $R / \gamma^{3}$ between the particles corresponding to small retarded angles $\gamma u \ll 1$ (see (5)). In this case the acceleration term and renormalized Coulomb term of eq. (7) take the form:

$$
\begin{aligned}
& \frac{e^{2}}{R^{2}}\left[\frac{\frac{u^{2}}{8}-(1-\beta)}{2\left(\frac{u^{2}}{8}+1-\beta\right)^{3}}\right] \simeq-\frac{2 e^{2} \gamma^{4}}{R^{2}} \\
& \frac{e^{2}}{R^{2} \gamma^{2} u^{2}}\left[\frac{\frac{3 u^{2}}{8}+1-\beta}{\left(\frac{u^{2}}{8}+1-\beta\right)^{3}}-\frac{1}{\left(\frac{u^{2}}{24}+1-\beta\right)^{2}}\right] \simeq \frac{2 e^{2} \gamma^{4}}{3 R^{2}}
\end{aligned}
$$

The total radiative force acting on the front electron from the back one is decelerative and is given by

$$
\left(\frac{d \mathcal{E}}{d(c t)}\right)_{C S R} \simeq-\frac{4 e^{2} \gamma^{4}}{3 R^{2}} \quad \text { at } \quad\left(s-s^{\prime}\right) \ll \frac{R}{\gamma^{3}} .
$$

To understand this result one should take into account that for each electron the rate of energy loss due to the radiative self-action is equal to

$$
\left(\frac{d \mathcal{E}}{d(c t)}\right)_{S R}=\left(\frac{d \mathcal{E}^{\prime}}{d(c t)}\right)_{S R}=-\frac{2 e^{2} \gamma^{4}}{3 R^{2}}
$$

As a result, the total rate of energy loss for the system of two electrons is equal to

$$
\left(\frac{d\left(\mathcal{E}+\mathcal{E}^{\prime}\right)}{d(c t)}\right)_{t o t} \simeq-\frac{8 e^{2} \gamma^{4}}{3 R^{2}} \quad \text { at } \quad\left(s-s^{\prime}\right) \ll \frac{R}{\gamma^{3}} .
$$


So, we have obtained a well-known result which states that the system of two particles at small distances between them radiates as one particle with double charge. This result indicates that the renormalization procedure (7) has been performed correctly. Indeed, this correct result could not be obtained without taking into account the renormalized Coulomb field which is independent of acceleration.

Let us consider now the asymptote of a large distance between the particles $\left(s-s^{\prime}\right) \gg R / \gamma^{3}$ corresponding to large retarded angles $u \gg 1 / \gamma$. In this case the renormalized Coulomb term is negligible and we have:

$$
\left(\frac{d \mathcal{E}}{d(c t)}\right)_{C S R} \simeq \frac{32 e^{2}}{R^{2} u^{4}} \quad \text { at } \quad \gamma u \gg 1
$$

Using eq. (5) we can express the retarded angle in the terms of the distance between the electrons

$$
u \simeq\left[\frac{24\left(s-s^{\prime}\right)}{R}\right]^{1 / 3}
$$

and finally get [12]:

$$
\left(\frac{d \mathcal{E}}{d(c t)}\right)_{C S R} \simeq \frac{2 e^{2}}{3^{4 / 3} R^{2 / 3}\left(s-s^{\prime}\right)^{4 / 3}} \quad \text { at } \quad\left(s-s^{\prime}\right) \gg \frac{R}{\gamma^{3}}
$$

In the general case the rate of the energy change of the front electron under the action of the radiative force from the side of the back electron can be written in the following form:

$$
\left(\frac{d \mathcal{E}}{d(c t)}\right)_{C S R}=-\frac{e^{2} \gamma^{4}}{R^{2}} \Phi\left(\hat{s}-\hat{s}^{\prime}\right)
$$

where function $\Phi\left(\hat{s}-\hat{s}^{\prime}\right)$ is given by the formula:

$$
\Phi=-\frac{\hat{u}^{2}-4}{2\left(\frac{\hat{u}^{2}}{4}+1\right)^{3}}-\frac{4}{\hat{u}^{2}}\left[\frac{\frac{3 \hat{u}^{2}}{4}+1}{\left(\frac{\hat{u}^{2}}{4}+1\right)^{3}}-\frac{1}{\left(\frac{\hat{u}^{2}}{12}+1\right)^{2}}\right] .
$$

The normalized retarded angle $\hat{u}=\gamma u$ is the function of normalized distance between electrons $\left(\hat{s}-\hat{s}^{\prime}\right)=\left(s-s^{\prime}\right) \gamma^{3} / R$ and can be obtained by solving the cubic equation

$$
\left(\hat{s}-\hat{s}^{\prime}\right)=\frac{\hat{u}}{2}+\frac{\hat{u}^{3}}{24} .
$$


The plot of the function $\Phi\left(\hat{s}-\hat{s}^{\prime}\right)$ is presented in Fig. 1 (see also ref.[13]). It is seen from this plot that the radiative interaction force provides deceleration of the front electron at a small distance between the electrons, but at

$$
\left(s-s^{\prime}\right)=1.8 \frac{R}{\gamma^{3}},
$$

the radiative force changes the sign.

\section{Coherent radiation of an electron bunch moving on a circle}

In this section we study CSR of the electron bunch executing a circular motion. Using physically transparent method for the calculation of the radiation force, we extend the results obtained in refs. $[5,8-13]$ to the case when lower limitation in eq. (1) is not satisfied.

We begin with the case of rotating electron ring with homogeneous linear density $\lambda(s)=\lambda_{0}=$ const. Here and below it is assumed that $\lambda_{0} R / \gamma^{3} \gg 1$. The rate of the energy change of the reference particle with coordinate $s$ is given by the superposition of the radiation forces of the back particles:

$$
\left(\frac{d \mathcal{E}}{d(c t)}\right)_{C S R}=\int_{-\infty}^{s} d s^{\prime} \lambda\left(s^{\prime}\right) K\left(s-s^{\prime}\right)=\lambda_{0} \int_{-\infty}^{s} d s^{\prime} K\left(s-s^{\prime}\right)
$$

where $K\left(s-s^{\prime}\right)$ is the rate of the energy change of the reference particle due to the action of the back particle with coordinate $s^{\prime}$ and $\lambda\left(s^{\prime}\right) d s^{\prime}=d N$ is number of particles inside interval $d s^{\prime}$. The kernel $K\left(s-s^{\prime}\right)$ is given by eq. (7) with the retarded angle $u$ given by eq. (5) as function of $\left(s-s^{\prime}\right)$. For the further consideration it is more convenient to go over to the retarded angle as independent variable. The expression for the Jacobian of transformation can be obtained from eq. (5):

$$
\frac{d\left(s-s^{\prime}\right)}{d u}=(1-\beta) R+\frac{R u^{2}}{8}
$$

As a result, eq. (14) takes the form:

$$
\left(\frac{d \mathcal{E}}{d(c t)}\right)_{C S R}=\frac{2 e^{2} \gamma \lambda_{0}}{R} \int_{0}^{\infty} d(\gamma u)\left[1+\frac{\gamma^{2} u^{2}}{4}\right] \times
$$




$$
\left\{\frac{\frac{\gamma^{2} u^{2}}{4}-1}{2\left(\frac{\gamma^{2} u^{2}}{4}+1\right)^{3}}+\frac{1}{\gamma^{2} u^{2}}\left[\frac{\frac{3 \gamma^{2} u^{2}}{4}+1}{\left(\frac{\gamma^{2} u^{2}}{4}+1\right)^{3}}-\frac{1}{\left(\frac{\gamma^{2} u^{2}}{12}+1\right)^{2}}\right]\right\}
$$

It should be noticed that setting the integration limit equal to infinity in eq. (14) and (15) is justified by ultrarelativistic approximation when the integral over a region of small angles $u \ll 1$ gives the main contribution. Integrating eq. (14) we obtain well-known result that uniformly charged ring does not radiate:

$$
\begin{gathered}
\left(\frac{d \mathcal{E}}{d(c t)}\right)_{C S R}=-\frac{4 e^{2} \gamma \lambda_{0}}{R}\left\{\lim _{\gamma u \rightarrow \infty}\left[\frac{(\gamma u)\left(8+\gamma^{2} u^{2}\right)}{\left(4+\gamma^{2} u^{2}\right)\left(12+\gamma^{2} u^{2}\right)}\right]-\right. \\
\left.\lim _{\gamma u \rightarrow 0}\left[\frac{(\gamma u)\left(8+\gamma^{2} u^{2}\right)}{\left(4+\gamma^{2} u^{2}\right)\left(12+\gamma^{2} u^{2}\right)}\right]\right\}=0 .
\end{gathered}
$$

This result could be also derived without using ultrarelativistic approximation. Starting with Lienard-Wiechert fields one can show that system of $N$ identical equidistant charges $q$ moving with constant velocity $v$ along an arbitrary closed path does not radiate in the limit of $N \rightarrow \infty$ and $N q=$ const, and the electric and magnetic fields of the system are the usual static values (see, for example, the book of Jackson [15]). We will use this important result in section 5 to simplify calculations.

Now let us consider the case of the electron bunch with an arbitrary distribution of the linear density satisfying the following condition:

$$
\frac{R}{\gamma^{3}} \frac{d \lambda(s)}{d s} \ll \lambda(s)
$$

This approximation means that characteristic length of the bunch is much larger than $R / \gamma^{3}$. To calculate integral

$$
\left(\frac{d \mathcal{E}}{d(c t)}\right)_{C S R}=\int_{-\infty}^{s} d s^{\prime} \lambda\left(s^{\prime}\right) K\left(s-s^{\prime}\right)
$$

one should take into account the property of the kernel $K^{\prime}\left(s-s^{\prime}\right)$ :

$$
\int_{-\infty}^{s} d s^{\prime} K\left(s-s^{\prime}\right)=0
$$

which has been proved above. Using eqs. (17) and (19) one can simplify eq. (18) 
in the following way. The integral (18) is written down as a sum of two integrals

$$
\int_{-\infty}^{s} d s^{\prime} \lambda\left(s^{\prime}\right) K\left(s-s^{\prime}\right)=\int_{-\infty}^{s-\delta} d s^{\prime} \lambda\left(s^{\prime}\right) K\left(s-s^{\prime}\right)+\int_{s-\delta}^{s} d s^{\prime} \lambda\left(s^{\prime}\right) K\left(s-s^{\prime}\right)
$$

where $\delta$ satisfies the following conditions:

$$
R / \gamma^{3} \ll \delta, \quad \delta \frac{d \lambda(s)}{d s} \ll \lambda(s)
$$

Using the first condition of eq. (21), we replace the rigorous expression for $K\left(s-s^{\prime}\right)$ by asymptotical expression (11) in the first integral of eq. (20):

$$
\int_{-\infty}^{s-\delta} d s^{\prime} \lambda\left(s^{\prime}\right) K\left(s-s^{\prime}\right) \simeq \frac{2 e^{2}}{3^{4 / 3} R^{2 / 3}} \int_{-\infty}^{s-\delta} d s^{\prime} \frac{\lambda\left(s^{\prime}\right)}{\left(s-s^{\prime}\right)^{4 / 3}} .
$$

Using the second condition of eq. (21), we calculate the second integral of eq. (20):

$$
\int_{s-\delta}^{s} d s^{\prime} \lambda\left(s^{\prime}\right) K\left(s-s^{\prime}\right) \simeq \lambda(s) \int_{s-\delta}^{s} d s^{\prime} K\left(s-s^{\prime}\right) .
$$

Then, using the property (19) of the kernel $K\left(s-s^{\prime}\right)$, it is transformed to:

$$
\int_{s-\delta}^{s} d s^{\prime} \lambda\left(s^{\prime}\right) K\left(s-s^{\prime}\right) \simeq-\lambda(s) \int_{-\infty}^{s-\delta} d s^{\prime} K\left(s-s^{\prime}\right)
$$

Then the asymptote of eq. (11) is used:

$$
\int_{s-\delta}^{s} d s^{\prime} \lambda\left(s^{\prime}\right) K\left(s-s^{\prime}\right) \simeq-\frac{2 e^{2} \lambda(s)}{3^{4 / 3} R^{2 / 3}} \int_{-\infty}^{s-\delta} \frac{d s^{\prime}}{\left(s-s^{\prime}\right)^{4 / 3}}
$$

and after integration by parts we obtain the result of integration of eq. (18):

$$
\begin{aligned}
& \left(\frac{d \mathcal{E}}{d(c t)}\right)_{C S R}=\int_{-\infty}^{s} d s^{\prime} \lambda\left(s^{\prime}\right) K\left(s-s^{\prime}\right) \simeq \\
& \frac{2 e^{2}}{3^{4 / 3} R^{2 / 3}}\left[\int_{-\infty}^{s-\delta} d s^{\prime} \frac{\lambda\left(s^{\prime}\right)}{\left(s-s^{\prime}\right)^{4 / 3}}-\lambda(s) \int_{-\infty}^{s-\delta} \frac{d s^{\prime}}{\left(s-s^{\prime}\right)^{4 / 3}}\right]=
\end{aligned}
$$




$$
-\frac{2 e^{2}}{3^{1 / 3} R^{2 / 3}} \int_{-\infty}^{s-\delta} \frac{d s^{\prime}}{\left(s-s^{\prime}\right)^{1 / 3}} \frac{d \lambda\left(s^{\prime}\right)}{d s^{\prime}} \simeq-\frac{2 e^{2}}{3^{1 / 3} R^{2 / 3}} \int_{-\infty}^{s} \frac{d s^{\prime}}{\left(s-s^{\prime}\right)^{1 / 3}} \frac{d \lambda\left(s^{\prime}\right)}{d s^{\prime}}
$$

At the last step of transformation of eq. (22) the second condition (21) has been used.

For the first time formula (22) has been derived in ref. [12]. It can be used for the calculation of the total CSR power $P$. For periodical circular motion one can write:

$$
P=-\int_{-\infty}^{\infty} d s \lambda(s)\left(\frac{d \mathcal{E}(s)}{d(c t)}\right)_{C S R}
$$

Let us consider two frequently used models of the density distribution. In the case of Gaussian distribution:

$$
\lambda(s)=\frac{N}{(2 \pi)^{1 / 2} \sigma} \exp \left[-\frac{s^{2}}{2 \sigma^{2}}\right]
$$

it is easy to obtain $[5,12,13]$ :

$$
\left(\frac{d \mathcal{E}}{d(c t)}\right)_{C S R} \simeq-\frac{2 N e^{2}}{(2 \pi)^{1 / 2} 3^{1 / 3} R^{2 / 3} \sigma^{4 / 3}} F\left(\frac{s}{\sigma}\right)
$$

where the function $F$ is given with the expression:

$$
F(\xi)=\int_{-\infty}^{\xi} \frac{d \xi^{\prime}}{\left(\xi-\xi^{\prime}\right)^{1 / 3}} \frac{d}{d \xi^{\prime}} e^{-\left(\xi^{\prime}\right)^{2} / 2}
$$

In accordance with eq. (23) total CSR power is equal to

$$
P \simeq \frac{2^{4 / 3} 3^{1 / 6} N^{2} e^{2}}{\pi R^{2 / 3} \sigma^{4 / 3}}\left[\Gamma\left(\frac{2}{3}\right)\right]^{2}
$$

For a stepped density distribution (i.e. at $\lambda(s)=N / l_{b}=$ const in interval $\left.0<s<l_{b}\right)$ one can obtain $[5,11,12]$ :

$$
\begin{aligned}
& \left(\frac{d \mathcal{E}}{d(c t)}\right)_{C S R} \simeq-\frac{2 N e^{2}}{3^{1 / 3} R^{2 / 3} l_{b} s^{1 / 3}}, \\
& P \simeq \frac{3^{2 / 3} N^{2} e^{2}}{R^{2 / 3} l_{b}^{4 / 3}} .
\end{aligned}
$$


The results (26) and (28) are in a full agreement with the results of calculations of CSR power in far zone [8-10].

Let us perform a more detailed study of the case of the stepped bunch profile. It is seen that eq. (27) contains singularity at $s \Rightarrow 0$, i.e. the rate of the energy loss of the tail particles becomes infinitely large. Nevertheless, this region of parameters is out of applicability region given by condition (17). Under this condition one has to consider the stepped profile in such a way that at the edges of the bunch there should be a smooth decrease of the bunch density at characteristic length which is small with respect to the bunch length, but large with respect to $R / \gamma^{3}$.

Using formalism developed in this paper we can obtain rigorous solution for the case of the stepped bunch profile which applicability region does not limited with the above mentioned restriction. Indeed, the bunch with the stepped density profile constitutes a fraction of a homogeneous electron ring. So, one can use expressions (15) and (16) where the infinity limit of integration should be replaced by finite value of the retarded angle. As a result, one obtains:

$$
\left(\frac{d \mathcal{E}}{d(c t)}\right)_{C S R}=-\frac{4 e^{2} N \gamma}{R l_{b}} \frac{\left(\gamma u_{s}\right)\left(8+\gamma^{2} u_{s}^{2}\right)}{\left(4+\gamma^{2} u_{s}^{2}\right)\left(12+\gamma^{2} u_{s}^{2}\right)}
$$

where $u_{s}$ can be calculated using relation

$$
s=(1-\beta) R u_{s}+\frac{R u_{s}^{3}}{24} .
$$

It is seen that eq. (29) does not contain singularity at $s \Rightarrow 0$. It is interesting to study asymptotical behaviour of this function. In the limit of $s \gg R / \gamma^{3}$ corresponding to large retarded angles $\gamma u_{s} \gg 1$, one can obtain:

$$
\begin{aligned}
& u_{s} \simeq\left[\frac{24 s}{R}\right]^{1 / 3} \\
& \left(\frac{d \mathcal{E}}{d(c t)}\right)_{C S R} \simeq-\frac{4 N e^{2}}{R u_{s} l_{b}}=-\frac{2 N e^{2}}{3^{1 / 3} R^{2 / 3} l_{b} s^{1 / 3}} .
\end{aligned}
$$

This result is identical to eq. (27), but now one has the possibility to check the accuracy of this asymptote using eq. (29).

In the limit of $s \ll R / \gamma^{3}$ (i.e. at $\gamma u_{s} \ll 1$ ), it is easy to obtain:

$$
u_{s} \simeq \frac{s}{(1-\beta) R} \simeq \frac{2 \gamma^{2} s}{R},
$$




$$
\left(\frac{d \mathcal{E}}{d(c t)}\right)_{C S R} \simeq-\frac{4 \gamma^{4} N e^{2} s}{3 R^{2} l_{b}}
$$

After integration of eq. (23) over the bunch length $l_{b}$, the total radiation power of the bunch can be obtained in the limit of $l_{b} \ll R / \gamma^{3}$ :

$$
P \simeq \frac{2 N^{2} e^{2} \gamma^{4}}{3 R^{2}}
$$

It is seen, that a short bunch with $l_{b} \ll R / \gamma^{3}$ radiates as a single particle with the charge of $\mathrm{Ne}$ and the radiation power does not depend on the bunch length $l_{b}$.

\section{Radiative interaction of two electrons passing a bending magnet}

In this section we study the problem of radiative interaction of two electrons passing a bending magnet with curvature radius $R$. Electrons move with velocity $v$ along identical trajectories one after another (see Figs. 2 and 3 ). We assume the bending angle of the electron trajectory in the magnet $\phi_{m}$ to be small, $\phi_{m} \ll 1$. The field at the point $P$ of the front electron's location is determined with the retarded position $P^{\prime}$ of the back electron. It is evident that the radiative action of the back electron on the first one can take place only in two parts of the trajectory, namely in the arc (inside the magnet) and on the straight line after the magnet. Four cases of radiative interaction should be considered:

A: The point $P$ is in the arc at azimuthal position $\phi(\phi=0$ corresponds to the entrance into the magnet). If the distance between electrons $\left(s-s^{\prime}\right)$ satisfies the condition

$$
\left(s-s^{\prime}\right)>(1-\beta) R \phi+\frac{R \phi^{3}}{24},
$$

then the point $P^{\prime}$ is on the straight line before the magnet (see Fig. 3a).

B: The point $P$ is in the arc and the distance between electrons satisfies the condition

$$
\left(s-s^{\prime}\right)<(1-\beta) R \phi+\frac{R \phi^{3}}{24} .
$$

In this case the point $P^{\prime}$ is in the arc, too (see Fig. $3 \mathrm{~b}$ ). 
C: The point $P$ is on the straight line after magnet at a distance $x$ from the exit of the magnet and the distance between the electrons is within the limits of

$$
\left(s-s^{\prime}\right)>(1-\beta)\left(R \phi_{m}+x\right)+\frac{R \phi_{m}^{3}}{24} \frac{R \phi_{m}+4 x}{R \phi_{m}+x} .
$$

In this case the point $P^{\prime}$ is on the straight line before the magnet (see Fig. 3c).

D: The point $P$ is on the straight line after the magnet. When

$$
\left(s-s^{\prime}\right)<(1-\beta)\left(R \phi_{m}+x\right)+\frac{R \phi_{m}^{3}}{24} \frac{R \phi_{m}+4 x}{R \phi_{m}+x},
$$

the point $P^{\prime}$ is in the arc (see Fig. $3 \mathrm{~d}$ ).

The procedure for calculation of the energy change of the front electron due to the radiative action of the back electron is identical to that described in section 2 .

For the case (A) we have only renormalized Coulomb term, leading to the radiative interaction:

$$
\begin{aligned}
\left(\frac{d \mathcal{E}}{d(c t)}\right)_{A}= & \frac{4 e^{2} \gamma^{4}}{R^{2}}(\hat{\phi}+\hat{y})^{2}\left\{\frac{(\hat{\phi}+\hat{y})^{2}+\hat{\phi}^{3}\left(\frac{3 \hat{\phi}}{4}+\hat{y}\right)}{\left[(\hat{\phi}+\hat{y})^{2}+\frac{\hat{\phi}^{4}}{4}\right]^{3}}-\right. \\
& \left.\frac{1}{\left[(\hat{\phi}+\hat{y})^{2}+\frac{\hat{\phi}^{3}}{12}(\hat{\phi}+4 \hat{y})\right]^{2}}\right\},
\end{aligned}
$$

where $\hat{\phi}=\gamma \phi, \hat{y}=y \gamma / R$ and $y$ is the distance between the point $P^{\prime}$ and the entrance to the magnet (see Fig. 3a). The normalized distance $\hat{y}$ is the root of the equation:

$$
\left(\hat{s}-\hat{s}^{\prime}\right)=\frac{\hat{\phi}+\hat{y}}{2}+\frac{\hat{\phi}^{3}}{24} \frac{\hat{\phi}+4 \hat{y}}{\hat{\phi}+\hat{y}},
$$

where $\left(\hat{s}-\hat{s}^{\prime}\right)=\left(s-s^{\prime}\right) \gamma^{3} / R$.

For the case (B) eq. (7) can be used: 


$$
\begin{aligned}
\left(\frac{d \mathcal{E}}{d(c t)}\right)_{B}= & \frac{4 e^{2} \gamma^{4}}{R^{2}}\left\{\frac{\frac{\hat{u}^{2}}{4}-1}{2\left(1+\frac{\hat{u}^{2}}{2}\right)^{3}}+\right. \\
& \left.\frac{1}{\hat{u}^{2}}\left[\frac{1+\frac{3 \hat{u}^{2}}{4}}{\left(1+\frac{\hat{u}^{2}}{4}\right)^{3}}-\frac{1}{\left(1+\frac{\hat{u}^{2}}{12}\right)^{2}}\right]\right\} .
\end{aligned}
$$

Here we have introduced the notion of the relative normalized azimuthal position $\hat{u}=\hat{\phi}-\hat{\phi}^{\prime}$ between the points $P$ and $P^{\prime}$ which is the root of equation:

$$
\left(\hat{s}-\hat{s}^{\prime}\right)=\frac{\hat{u}}{2}+\frac{\hat{u}^{3}}{24} \text {. }
$$

Case (B) can be referred as a steady-state regime, because the radiative force does not depend on the azimuthal position $\hat{\phi}$ of the point $P$.

For the case $(\mathrm{C})$ we have only the renormalized Coulomb term:

$$
\begin{aligned}
\left(\frac{d \mathcal{E}}{d(c t)}\right)_{C}= & \frac{4 e^{2} \gamma^{4}}{R^{2}}\left(\hat{\phi}_{m}+\hat{x}+\hat{y}\right)^{2} \times \\
& \left\{\frac{\left(\hat{\phi}_{m}+\hat{x}+\hat{y}\right)^{2}+\hat{\phi}_{m}^{2}\left(\frac{3 \hat{\phi}_{m}^{2}}{4}+\hat{x}^{2}+2 \hat{x} \hat{\phi}_{m}+\hat{y} \hat{\phi}_{m}+2 \hat{x} \hat{y}\right)}{\left[\left(\hat{\phi}_{m}+\hat{x}+\hat{y}\right)^{2}+\frac{\hat{\phi}_{m}^{2}}{4}\left(\hat{\phi}_{m}+2 \hat{x}\right)^{2}\right]^{3}}-\right. \\
& \left.\frac{1}{\left[\left(\hat{\phi}_{m}+\hat{x}+\hat{y}\right)^{2}+\frac{\hat{\phi}_{m}^{2}}{12}\left(\hat{\phi}_{m}^{2}+4 \hat{x} \hat{\phi}_{m}+4 \hat{y} \hat{\phi}_{m}+12 \hat{x} \hat{y}\right)\right]^{2}}\right\}
\end{aligned}
$$

where $\hat{\phi}_{m}=\gamma \phi_{m}, \hat{x}=x \gamma / R$ and $\hat{y}=y \gamma / R$. To find $\hat{y}$ one has to solve the equation:

$$
\left(\hat{s}-\hat{s}^{\prime}\right)=\frac{\hat{\phi}_{m}+\hat{x}+\hat{y}}{2}+\frac{\hat{\phi}_{m}^{2}}{24} \frac{\hat{\phi}_{m}^{2}+4 \hat{\phi}_{m}(\hat{x}+\hat{y})+12 \hat{x} \hat{y}}{\hat{\phi}_{m}+\hat{x}+\hat{y}} .
$$

The following expression corresponds to the case (D):

$$
\left(\frac{d \mathcal{E}}{d(c t)}\right)_{D}=\frac{4 e^{2} \gamma^{4}}{R^{2}}(\hat{\psi}+\hat{x})^{2} \times
$$




$$
\begin{aligned}
& \left\{\frac{\hat{\psi}^{2}\left[\frac{\hat{\psi}^{2}}{4}(\hat{\psi}+2 \hat{x})^{2}-(\hat{\psi}+\hat{x})^{2}\right]}{2\left[(\hat{\psi}+\hat{x})^{2}+\frac{\hat{\psi}^{2}}{4}(\hat{\psi}+2 \hat{x})^{2}\right]^{3}}+\right. \\
& \frac{(\hat{\psi}+\hat{x})^{2}+\hat{\psi}^{2}\left(\frac{3 \hat{\psi}^{2}}{4}+\hat{x}^{2}+2 \hat{x} \hat{\psi}\right)}{\left[(\hat{\psi}+\hat{x})^{2}+\frac{\hat{\psi}^{2}}{4}(\hat{\psi}+2 \hat{x})^{2}\right]^{3}}- \\
& \left.\frac{1}{\left[(\hat{\psi}+\hat{x})^{2}+\frac{\hat{\psi}^{2}}{12}\left(\hat{\psi}^{2}+4 \hat{x} \hat{\psi}\right)\right]^{2}}\right\}
\end{aligned}
$$

Here $\hat{\psi}=\hat{\phi}_{m}-\hat{\phi}^{\prime}$ and $\hat{\phi}^{\prime}=\gamma \phi^{\prime}$ is normalized azimuth of the point $P^{\prime}$. The value of $\hat{\psi}$ can be found by solving the equation:

$$
\left(\hat{s}-\hat{s}^{\prime}\right)=\frac{\hat{\psi}+\hat{x}}{2}+\frac{\hat{\psi}^{2}}{24} \frac{\hat{\psi}^{2}+4 \hat{x} \hat{\psi}}{\hat{\psi}+\hat{x}},
$$

The expression for the radiative force presented here can be used as a basis for calculations of the radiative interaction of the particles inside the bunch of any length and arbitrary density distribution. As far as we know, the only density profile allowing rigorous analytical solution is a stepped one. This case is studied in detail in the next section. For calculation of a general case this set of formulae could be implemented in numerical codes.

\section{Coherent radiation of a rectangular bunch passing a bending magnet}

In this section we consider CSR effects of the electron bunch of any length with rectangular density distribution passing a bending magnet.

First, we consider the case of infinitely long electron bunch with the current $I=e v \lambda_{0}=$ const. A current circuit consists of the arc and semi-infinite straight lines (see Fig. 2). The angle between the straight lines is equal to the bending angle $\phi_{m}$ of the magnet. We will show that radiative interaction force is equal to zero at any point of such a circuit. This fact is important for the following investigation of the CSR effects for the bunch of finite length.

Let us find the rate of the energy change for a reference particle at some point $P$ in the arc of the magnet. Let the azimuth of this point be equal to $\phi$. Using formula (33) and calculating Jacobian of transformation $\left(\hat{s}-\hat{s}^{\prime}\right) \Rightarrow \hat{y}$ with the 
help of eq. (34) one can write the superposition of radiative interaction forces for the source points $P^{\prime}$ located before the entrance into the magnet:

$$
\begin{aligned}
\left(\frac{d \mathcal{E}}{d(c t)}\right)_{A}= & \frac{2 e^{2} \gamma \lambda_{0}}{R} \int_{0}^{\infty} d \hat{y}\left\{\frac{(\hat{\phi}+\hat{y})^{2}+\hat{\phi}^{3}\left(\frac{3 \hat{\phi}}{4}+\hat{y}\right)}{\left[(\hat{\phi}+\hat{y})^{2}+\frac{\hat{\phi}^{4}}{4}\right]^{2}}-\right. \\
& \left.\frac{(\hat{\phi}+\hat{y})^{2}+\frac{\hat{\phi}^{4}}{4}}{\left[(\hat{\phi}+\hat{y})^{2}+\frac{\hat{\phi}^{3}}{12}(\hat{\phi}+4 \hat{y})\right]^{2}}\right\} .
\end{aligned}
$$

The integration of this expression gives the result:

$$
\begin{aligned}
\left(\frac{d \mathcal{E}}{d(c t)}\right)_{A}= & \frac{4 e^{2} \gamma \lambda_{0}}{R}\left\{\frac{\hat{\phi}^{2}+2}{\hat{\phi}\left(\hat{\phi}^{2}+4\right)}-\frac{6}{\hat{\phi}\left(\hat{\phi}^{2}+12\right)}\right\}= \\
& \frac{4 e^{2} \gamma \lambda_{0}}{R} \frac{\hat{\phi}\left(8+\hat{\phi}^{2}\right)}{\left(4+\hat{\phi}^{2}\right)\left(12+\hat{\phi}^{2}\right)} .
\end{aligned}
$$

To calculate the radiative interaction force acting on the reference particle from the back particles with retarded positions $P^{\prime}$ inside the arc, one should use eq. (35) and (36):

$$
\begin{aligned}
\left(\frac{d \mathcal{E}}{d(c t)}\right)_{B}= & \frac{2 e^{2} \gamma \lambda_{0}}{R} \int_{0}^{\hat{\phi}} d \hat{u}\left(1+\frac{\hat{u}^{2}}{4}\right)\left\{\frac{\frac{\hat{u}^{2}}{4}-1}{2\left(1+\frac{\hat{u}^{2}}{4}\right)^{3}}+\right. \\
& \left.\frac{1}{\hat{u}^{2}}\left[\frac{1+\frac{3 \hat{u}^{2}}{4}}{\left(1+\frac{\hat{u}^{2}}{4}\right)^{3}}-\frac{1}{\left(1+\frac{\hat{u}^{2}}{12}\right)^{2}}\right]\right\}= \\
& -\frac{4 e^{2} \gamma \lambda_{0}}{R} \frac{\hat{\phi}\left(8+\hat{\phi}^{2}\right)}{\left(4+\hat{\phi}^{2}\right)\left(12+\hat{\phi}^{2}\right)} .
\end{aligned}
$$

It is seen that total energy change of the reference particle due to the radiative interaction with the back particles is equal to zero:

$$
\left(\frac{d \mathcal{E}}{d(c t)}\right)_{t o t}=\left(\frac{d \mathcal{E}}{d(c t)}\right)_{A}+\left(\frac{d \mathcal{E}}{d(c t)}\right)_{B}=0 .
$$


Now let the reference point $P$ to be placed on the straight line after the magnet at some distance $x$ from the magnet exit. The action of the particles with retarding positions $P^{\prime}$ on the straight line before the magnet is calculated using eq. (37) and (38):

$$
\begin{aligned}
& \left(\frac{d \mathcal{E}}{d(c t)}\right)_{C}=\frac{2 e^{2} \gamma \lambda_{0}}{R} \int_{0}^{\infty} d \hat{y} \times \\
& \left\{\begin{array}{c}
\frac{\left(\hat{\phi}_{m}+\hat{x}+\hat{y}\right)^{2}+\hat{\phi}_{m}^{2}\left(\frac{3 \hat{\phi}_{m}^{2}}{4}+\hat{x}^{2}+2 \hat{x} \hat{\phi}_{m}+\hat{y} \hat{\phi}_{m}+2 \hat{x} \hat{y}\right)}{\left[\left(\hat{\phi}_{m}+\hat{x}+\hat{y}\right)^{2}+\frac{\hat{\phi}_{m}^{2}}{4}\left(\hat{\phi}_{m}+2 \hat{x}\right)^{2}\right]^{2}}- \\
\left.\frac{\left(\hat{\phi}_{m}+\hat{x}+\hat{y}\right)^{2}+\frac{\hat{\phi}_{m}^{2}}{4}\left(\hat{\phi}_{m}+2 \hat{x}\right)^{2}}{\left[\left(\hat{\phi}_{m}+\hat{x}+\hat{y}\right)^{2}+\frac{\hat{\phi}_{m}^{2}}{12}\left(\hat{\phi}_{m}^{2}+4 \hat{x} \hat{\phi}_{m}+4 \hat{y} \hat{\phi}_{m}+12 \hat{x} \hat{y}\right)\right]^{2}}\right\}
\end{array}\right.
\end{aligned}
$$

When retarded positions of the back particles $P^{\prime}$ are within the arc, the following expression can be written with the help of eq. (39) and (40):

$$
\begin{aligned}
\left(\frac{d \mathcal{E}}{d(c t)}\right)_{D}= & \frac{2 e^{2} \gamma \lambda_{0}}{R} \int_{0}^{\dot{\phi}_{m}} d \hat{\psi} \times \\
& \left\{\frac{\hat{\psi}^{2}\left[\frac{\hat{\psi}^{2}}{4}(\hat{\psi}+2 \hat{x})^{2}-(\hat{\psi}+\hat{x})^{2}\right]}{2\left[(\hat{\psi}+\hat{x})^{2}+\frac{\hat{\psi}^{2}}{4}(\hat{\psi}+2 \hat{x})^{2}\right]^{2}}+\right. \\
& \frac{(\hat{\psi}+\hat{x})^{2}+\hat{\psi}^{2}\left(\frac{3 \hat{\psi}^{2}}{4}+\hat{x}^{2}+2 \hat{x} \hat{\psi}\right)}{\left[(\hat{\psi}+\hat{x})^{2}+\frac{\dot{\psi}^{2}}{4}(\hat{\psi}+2 \hat{x})^{2}\right]^{2}}- \\
& \left.\frac{(\hat{\psi}+\hat{x})^{2}+\frac{\dot{\psi}^{2}}{4}(\hat{\psi}+2 \hat{x})^{2}}{\left[(\hat{\psi}+\hat{x})^{2}+\frac{\dot{\psi}^{3}}{12}(\hat{\psi}+4 \hat{x})\right]^{2}}\right\}
\end{aligned}
$$

After the integration one can write:

$$
\left(\frac{d \mathcal{E}}{d(c t)}\right)_{C}=-\left(\frac{d \mathcal{E}}{d(c t)}\right)_{D}=
$$




$$
\begin{aligned}
& \frac{4 e^{2} \gamma \lambda_{0}}{R}\left[\frac{2 \hat{x}\left(\hat{\phi}_{m}^{2}+1\right)+\hat{\phi}_{m}\left(\hat{\phi}_{m}^{2}+2\right)}{4 \hat{x}^{2}\left(\hat{\phi}_{m}^{2}+1\right)+4 \hat{x} \hat{\phi}_{m}\left(\hat{\phi}_{m}^{2}+2\right)+\hat{\phi}_{m}^{2}\left(\hat{\phi}_{m}^{2}+4\right)}-\right. \\
& \left.\frac{6\left(\hat{x}+\hat{\phi}_{m}\right)}{12 \hat{x}^{2}+4 \hat{x} \hat{\phi}_{m}\left(\hat{\phi}_{m}^{2}+6\right)+\hat{\phi}_{m}^{2}\left(\hat{\phi}_{m}^{2}+12\right)}\right] .
\end{aligned}
$$

So, we have obtained that there is no radiative interaction for the case of infinitely long electron bunch.

Now let us consider the bunch with stepped density profile and arbitrary length $l_{b}$. The total number of the particles in the bunch is equal to $N$ which corresponds to the linear density is equal to $\lambda_{0}=N / l_{b}$. We have to calculate the radiative force acting on a reference particle being placed at a distance $s$ from the bunch tail. It is useful to define parameter $\hat{\phi}_{s}$ as a solution of the cubic equation:

$$
\hat{s}=\frac{\hat{\phi}_{s}}{2}+\frac{\hat{\phi}_{s}^{3}}{24}
$$

Solving this equation one obtains

$$
\dot{\phi}_{s}=\sqrt[3]{12 \hat{s}+\sqrt{64+144 \hat{s}^{2}}}-\sqrt[3]{\sqrt{64+144 \hat{s}^{2}}-12 \hat{s}} .
$$

The electric field acting on the reference particle when it passes point $P$ is defined with the superposition of retarded electric fields of the back particles. Let the retarded position of the bunch tail be at point $P^{\prime}$ and consider possible scenario for location of the points $P$ and $P^{\prime}$. If the normalized angle of the arc $\hat{\phi}_{m}$ is larger than parameter $\hat{\phi}_{s}$, the configurations presented in Figs. 3a, $3 \mathrm{~b}$ and $3 \mathrm{~d}$ can take place. In the case when $\phi_{m}$ is less than $\hat{\phi}_{s}$, the Figs. 3a, $3 \mathrm{c}$ and $3 \mathrm{~d}$ represent possible configurations. Let us write the expressions for total energy change of the reference particle with coordinate $s$ :

$$
\begin{array}{ll}
\text { region } 1 \quad\left(\hat{\phi}_{s}<\hat{\phi}_{m}\right) \\
\Delta \hat{\mathcal{E}}=\int_{0}^{\hat{\phi}_{s}} d \hat{\phi} F_{a}(\hat{s}, \hat{\phi})+\int_{\hat{\phi}_{s}}^{\hat{\phi}_{m}} d \hat{\phi} F_{b}(\hat{s})+ \\
\int_{0}^{\hat{x}_{s}} d \hat{x} F_{d}(\hat{s}, \hat{x})
\end{array}
$$


region $2 \quad\left(\hat{\phi}_{m}<\hat{\phi}_{s}\right)$

$$
\begin{aligned}
& \Delta \hat{\mathcal{E}}=\int_{0}^{\hat{\phi}_{m}} d \hat{\phi} F_{a}(\hat{s}, \hat{\phi})+\int_{0}^{\hat{x}_{0}} d \hat{\phi} F_{c}(\hat{s}, \hat{x})+ \\
& \int_{\hat{x}_{0}}^{\hat{x}_{0}} d \hat{x} F_{d}(\hat{s}, \hat{x}) .
\end{aligned}
$$

Here

$$
\Delta \hat{\mathcal{E}}=\Delta \mathcal{E}\left(\frac{e^{2} N}{l_{b}}\right)^{-1}
$$

is the normalized energy loss, $F_{(a, b, c, d)}$ is normalized rate of the energy change along the trajectory and subscripts $(a, b, c, d)$ correspond to location of the points $P$ and $P^{\prime}$ in Figs. $3(a, b, c, d)$, respectively. The value of $x_{s}$ is defined as the distance between $P$ and $P^{\prime}$ at the moment when $P^{\prime}$ leaves the magnet (i.e. when it coincides with the point $B$ in Fig. 3). It can be simply shown that $x_{s}=s /(1-\beta)$ or, in normalized form $\hat{x}=2 \hat{s}$. The value of $x_{0}$ is equal to the distance $B P$ at that moment when $P^{\prime}$ enters the magnet (i.e. when it coincides with the point $A$ ). The normalized value of $\hat{x}_{0}$ can be found by solving the quadratic equation $(38)$ at $\hat{y}=0$. As a result, one can write:

$$
\hat{x}_{0}=\hat{s}-\hat{\phi}_{m}-\frac{\hat{\phi}_{m}^{3}}{6}+\frac{1}{6} \sqrt{\left(\hat{\phi}_{m}^{3}-6 \hat{s}\right)^{2}+9 \hat{\phi}_{m}^{4}} \text {. }
$$

The next problem is to calculate the functions $F_{a, b, c, d}$. The problem is significantly simplified if we remember that the radiative force tends to zero when retarded position of the bunch tail tends to infinity (see eq. (44) and (47)). To find $F_{a}(\hat{s}, \hat{\phi})$ one can use expressions $(41)$ and $(43)$, but with finite value for upper limit in eq. (41):

$$
\begin{aligned}
F_{a}(\hat{s}, \hat{\phi})= & 2 \int_{0}^{\hat{y}_{0}} d \hat{y}\left\{\frac{(\hat{\phi}+\hat{y})^{2}+\hat{\phi}^{3}\left(\frac{3 \hat{\phi}}{4}+\hat{y}\right)}{\left[(\hat{\phi}+\hat{y})^{2}+\frac{\hat{\phi}^{4}}{4}\right]^{2}}-\right. \\
& \left.\frac{(\hat{\phi}+\hat{y})^{2}+\frac{\hat{\phi}^{4}}{4}}{\left[(\hat{\phi}+\hat{y})^{2}+\frac{\hat{\phi}^{3}}{12}(\hat{\phi}+4 \hat{y})\right]^{2}}\right\}+ \\
& 2 \int_{0}^{\dot{\phi}} d \hat{u}\left(1+\frac{\hat{u}^{2}}{4}\right)\left\{\frac{\frac{\hat{u}^{2}}{4}-1}{2\left(1+\frac{\hat{u}^{2}}{4}\right)^{3}}+\right.
\end{aligned}
$$




$$
\left.\frac{1}{\hat{u}^{2}}\left[\frac{1+\frac{3 \hat{u}^{2}}{4}}{\left(1+\frac{\hat{u}^{2}}{4}\right)^{3}}-\frac{1}{\left(1+\frac{\hat{u}^{2}}{12}\right)^{2}}\right]\right\} \text {. }
$$

The upper limit of integration, $\hat{y}_{0}$, can be found by solving quadratic equation (34) and written as

$$
\hat{y}_{0}=\hat{s}-\hat{\phi}-\frac{\hat{\phi}^{3}}{6}+\frac{1}{6} \sqrt{\left(\hat{\phi}^{3}-6 \hat{s}\right)^{2}+9 \hat{\phi}^{4}} .
$$

Using eq. (44), the expression for $F_{a}$ can be written in the following simplified form:

$$
\begin{gathered}
F_{a}(\hat{s}, \hat{\phi})=-2 \int_{\hat{y}_{0}}^{\infty} d \hat{y}\left\{\frac{(\hat{\phi}+\hat{y})^{2}+\hat{\phi}^{3}\left(\frac{3 \hat{\phi}}{4}+\hat{y}\right)}{\left[(\hat{\phi}+\hat{y})^{2}+\frac{\hat{\phi}^{4}}{4}\right]^{2}}-\right. \\
\left.\frac{(\hat{\phi}+\hat{y})^{2}+\frac{\hat{\phi}^{4}}{4}}{\left[(\hat{\phi}+\hat{y})^{2}+\frac{\hat{\phi}^{3}}{12}(\hat{\phi}+4 \hat{y})\right]^{2}}\right\} .
\end{gathered}
$$

After the integration and substituting eq. (54) we obtain:

$$
F_{a}(\hat{s}, \hat{\phi})=-\frac{2}{\hat{\phi}}\left[1+\frac{\hat{\phi}^{3}-6 \hat{s}+3 \hat{\phi}}{\sqrt{\left(\hat{\phi}^{3}-6 \hat{s}\right)^{2}+9 \hat{\phi}^{4}}}\right]+\frac{1}{\hat{s}}
$$

The function $F_{b}(\hat{s})$ is given by expression (29):

$$
F_{b}(\hat{s})=-4 \frac{\hat{\phi}_{s}\left(\hat{\phi}_{s}^{2}+8\right)}{\left(\hat{\phi}_{s}^{2}+4\right)\left(\hat{\phi}_{s}^{2}+12\right)},
$$

and $\hat{\phi}_{s}$ is expressed in terms of $\hat{s}$ (see eq. (49)).

According to eq. (45) and (47) function $F_{c}$ can be written in the form:

$$
F_{c}(\hat{s}, \hat{x})=
$$




$$
\begin{aligned}
& -2 \int_{\hat{y}_{0}}^{\infty} d \hat{y}\left\{\frac{\left(\hat{\phi}_{m}+\hat{x}+\hat{y}\right)^{2}+\hat{\phi}_{m}^{2}\left(\frac{3 \hat{\phi}_{m}^{2}}{4}+\hat{x}^{2}+2 \hat{x} \hat{\phi}_{m}+\hat{y} \hat{\phi}_{m}+2 \hat{x} \hat{y}\right)}{\left[\left(\hat{\phi}_{m}+\hat{x}+\hat{y}\right)^{2}+\frac{\hat{\phi}_{m}^{2}}{4}\left(\hat{\phi}_{m}+2 \hat{x}\right)^{2}\right]^{2}}-\right. \\
& \left.\frac{\left(\hat{\phi}_{m}+\hat{x}+\hat{y}\right)^{2}+\frac{\hat{\phi}_{m}^{2}}{4}\left(\hat{\phi}_{m}+2 \hat{x}\right)^{2}}{\left[\left(\hat{\phi}_{m}+\hat{x}+\hat{y}\right)^{2}+\frac{\dot{\phi}_{m}^{2}}{12}\left(\hat{\phi}_{m}^{2}+4 \hat{x} \hat{\phi}_{m}+4 \hat{y} \hat{\phi}_{m}+12 \hat{x} \hat{y}\right)\right]^{2}}\right\}
\end{aligned}
$$

Here $\hat{y}_{0}$ is the solution of the quadratic equation (38) which can be rewritten in a more convenient form:

$$
\left(\hat{\phi}_{m}+\hat{x}+\hat{y}_{0}\right)^{2}+\left(\frac{\hat{\phi}_{m}^{3}}{3}+\hat{x} \hat{\phi}_{m}^{2}-2 \hat{s}\right)\left(\phi_{m}+\hat{x}+\hat{y}_{0}\right)-\frac{\hat{\phi}_{m}^{2}}{4}\left(\hat{\phi}_{m}+2 \hat{x}\right)^{2}=0 .
$$

Solving this equation we obtain:

$$
\begin{aligned}
\hat{y}_{0}= & \hat{s}-\hat{x}-\hat{\phi}_{m}-\frac{\hat{\phi}_{m}^{3}}{6}-\frac{\hat{x} \hat{\phi}_{m}^{2}}{2}+ \\
& \frac{1}{6} \sqrt{\left(\hat{\phi}^{3}+3 \hat{x} \hat{\phi}_{m}^{2}-6 \hat{s}\right)^{2}+9 \hat{\phi}_{m}^{2}\left(\hat{\phi}_{m}+2 \hat{x}\right)^{2}},
\end{aligned}
$$

and the final result for $F_{c}(\hat{s}, \hat{x})$ is

$$
\begin{aligned}
F_{c}(\hat{s}, \hat{x})= & -\frac{2}{\hat{\phi}_{m}+2 \hat{x}} \times \\
& {\left[1+\frac{\left(\hat{\phi}_{m}^{3}+3 \hat{x} \hat{\phi}_{m}^{2}-6 \hat{s}\right)+3\left(\hat{\phi}_{m}+2 \hat{x}\right)}{\sqrt{\left(\hat{\phi}_{m}^{3}+3 \hat{x} \hat{\phi}_{m}^{2}-6 \hat{s}\right)^{2}+9 \hat{\phi}_{m}^{2}\left(\hat{\phi}_{m}+2 \hat{x}\right)^{2}}}\right]+\frac{1}{\hat{s}} . }
\end{aligned}
$$

According to eq. (46) function $F_{d}(\hat{s}, \hat{x})$ is defined as follows:

$$
\begin{aligned}
F_{d}(\hat{s}, \hat{x})=2 & \int_{0}^{\hat{\psi}_{0}} d \hat{\psi}\left\{\frac{\hat{\psi}^{2}\left[\frac{\hat{\psi}^{2}}{4}(\hat{\psi}+2 \hat{x})^{2}-(\hat{\psi}+\hat{x})^{2}\right]}{2\left[(\hat{\psi}+\hat{x})^{2}+\frac{\hat{\psi}^{2}}{4}(\hat{\psi}+2 \hat{x})^{2}\right]^{2}}+\right. \\
& \frac{(\hat{\psi}+\hat{x})^{2}+\hat{\psi}^{2}\left(\frac{3 \dot{\psi}^{2}}{4}+\hat{x}^{2}+2 \hat{x} \hat{\psi}\right)}{\left[(\hat{\psi}+\hat{x})^{2}+\frac{\dot{\psi}^{2}}{4}(\hat{\psi}+2 \hat{x})^{2}\right]^{2}}-
\end{aligned}
$$




$$
\left.\frac{(\hat{\psi}+\hat{x})^{2}+\frac{\hat{\psi}^{2}}{4}(\hat{\psi}+2 \hat{x})^{2}}{\left[(\hat{\psi}+\hat{x})^{2}+\frac{\hat{\psi}^{3}}{12}(\hat{\psi}+4 \hat{x})\right]^{2}}\right\}
$$

where $\hat{\psi}_{0}$ is the solution of the quadratic equation (see eq. $(40)$ ):

$$
\hat{\psi}_{0}^{4}+4 \hat{x} \hat{\psi}_{0}^{3}+12 \hat{\psi}_{0}^{2}+24(\hat{x}-\hat{s}) \hat{\psi}_{0}+12 \hat{x}(\hat{x}-2 \hat{s})=0
$$

Using Descartes' rule of signs it is easy to check that this equation has only one positive real root. To express $\hat{\psi}_{0}$ in terms of $\hat{x}$ and $\hat{s}$ one can use, for instance, Ferrari's solution. Integral (61) can be replaced by complementary integral and could be calculated easily:

$$
F_{d}(\hat{s}, \hat{x})=-4\left[\frac{2 \hat{x}\left(\hat{\psi}_{0}^{2}+1\right)+\hat{\psi}_{0}\left(\hat{\psi}_{0}^{2}+2\right)}{4 \hat{x}^{2}\left(\hat{\psi}_{0}^{2}+1\right)+4 \hat{x} \hat{\psi}_{0}\left(\hat{\psi}_{0}^{2}+2\right)+\hat{\psi}_{0}^{2}\left(\hat{\psi}_{0}^{2}+4\right)}\right]+\frac{1}{\hat{s}} .
$$

Let us explain the origin of a simple form for the term $1 / \hat{s}$ appearing in the expressions (56), (60) and (63). It was proved in the beginning of this section that the radiative interaction force is equal to zero in the case of infinite circuit of the dc current. This property has been used when calculating the functions $F_{a}, F_{c}$ and $F_{d}$. The integral over trajectory from the retarded position of the bunch tail to the position of the reference particle was replaced by the integral (with opposite sign) over complementary semi-infinite imaginary circuit. The latter may even not coincide with real trajectory (an example is the calculation of function $F_{d}$ ). The advantage of such a method of calculation is explained by the fact that the integrand in complementary integral contains the only renormalized Coulomb term. The latter one consists of two terms and one of them, namely $e^{2} / \gamma^{2}\left(s-s^{\prime}\right)^{2}$, is integrated very simply (over $s^{\prime}$ from $s$ to infinity) and always gives the dependence $1 / s$ (see eq. $(56),(60)$ and $(63)$ ). The only complicated remaining procedure consists in the integration of the velocity term over additional semi-infinite circuit.

The same trick could be used for calculation of $F_{b}$ :

$$
F_{b}=-4 \frac{\hat{\phi}_{s}^{2}+2}{\hat{\phi}_{s}\left(\hat{\phi}_{s}^{2}+4\right)}+\frac{1}{\hat{s}}
$$

Taking into account eq. (48) one easily obtains (57). Finally, we should note that the method of complementary integral could be used efficiently in more complicated situations such as calculations of CSR effects in sequence of magnets, undulators etc. 
Formulae (56), (57), (60) and (63) present the normalized rate of the energy change for the particle with the reference coordinate $s$ in the bunch. The parts of trajectory, where these functions $F_{(a, b, c, d)}$ have to be used, are defined in eq. (50) and (51) as the corresponding limits of integration. To calculate the total energy change $\Delta \mathcal{E}$ of the reference particle one needs to perform integration in eqs. (50) and (51). The examples of numerical integration are presented in Figs. 4,5. Total energy loss of the whole bunch $\Delta \mathcal{E}_{\text {tot }}$ is obtained by integration of the energy loss of the reference particle over $s$ (in normalized notations):

$$
\Delta \hat{\mathcal{E}}_{\text {tot }}=\int_{0}^{\hat{\iota}_{b}} d \hat{s} \Delta \hat{\mathcal{E}}
$$

where

$$
\Delta \hat{\mathcal{E}}=\frac{l_{b}}{e^{2} N} \Delta \mathcal{E}, \quad \hat{l}_{b}=\frac{\gamma^{3}}{R} l_{b}, \quad \Delta \hat{\mathcal{E}}_{\text {tot }}=\frac{l_{b}^{2} \gamma^{3}}{e^{2} N^{2} R} \Delta \mathcal{E}_{t o t} .
$$

One can study the distribution of the total energy loss of the bunch along the trajectory:

$$
\frac{d \hat{\mathcal{E}}_{t o t}}{d \hat{z}}=\int_{0}^{\hat{l}_{b}} d \hat{s} \frac{d \hat{\mathcal{E}}}{d \hat{z}}
$$

where $\hat{z}$ is either $\hat{\phi}$ or $\hat{x}$ depending on the bunch position (inside or after the magnet, respectively), and $d \hat{\mathcal{E}} / d \hat{z}$ is equal to one of the functions $F_{(a, b, c, d)}$ depending on the particle position along the bunch $\hat{s}$. The angular position $\phi$, the position $x$ on the straight line after the magnet and the position $s$ of the particle in the bunch are normalized as follows:

$$
\hat{\phi}=\gamma \phi, \quad \hat{x}=\frac{\gamma}{R} x, \quad \hat{s}=\frac{\gamma^{3}}{R} s .
$$

\section{Analysis of the solutions}

In this section we perform analysis of asymptotics of the results obtained in the previous section for the electron bunch with rectangular density distribution. We introduce the notions of a "short" magnet (when $\hat{\phi}_{m} \ll 1$ ) and a "long" magnet $\left(\hat{\phi}_{m} \gg 1\right)$, a "short" bunch $\left(\hat{l}_{b} \ll 1\right)$ and a "long" bunch $\left(\hat{l}_{b} \gg 1\right)$. To 
compare "the length" of the bunch and the magnet we use the parameter $\hat{\phi}_{b}$ which is the solution of the cubic equation:

$$
\hat{l}_{b}=\frac{\hat{\phi}_{b}}{2}+\frac{\hat{\phi}_{b}^{3}}{24}
$$

When we write that the bunch is "longer" than the magnet, it means that $\hat{\phi}_{b}>\hat{\phi}_{m}$, and vice versa.

\section{1 "Short" magnet and bunch of arbitrary length}

This case is not only of theoretical interest. Magnets with $\hat{\phi}_{m} \ll 1$ can be used, for instance, as correctors in the beam transport systems. As for the bunch length, usually the condition $\hat{l}_{b} \gg 1$ is satisfied in practice. Let us consider, however, the bunch with arbitrary normalized length $\hat{l}_{b}$. This more common case could be useful for testing the numerical simulation codes. The case of $\hat{l}_{b} \gg 1$ will be considered more thoroughly. The functions $F_{(a, b, c, d)}$ are simplified at $\hat{\phi}_{m} \ll 1$ as follows:

$$
\begin{aligned}
& F_{a} \simeq-\frac{\hat{\phi}^{3}}{\hat{s}^{2}}\left(\frac{5}{12}-\frac{\hat{\phi}}{8 \hat{s}}\right), \\
& F_{b} \simeq-\frac{4 \hat{s}}{3}, \\
& F_{c} \simeq-\frac{\hat{\phi}_{m}^{3}}{\hat{s}^{2}}\left[\frac{5}{12}+\frac{\hat{x}}{\hat{\phi}_{m}}-\frac{\left(\hat{\phi}_{m}+2 \hat{x}\right)^{2}}{8 \hat{s} \hat{\phi}_{m}}\right], \\
& F_{d} \simeq-\frac{4 \hat{s}}{3}+\hat{x}+\frac{\hat{x}^{2}}{2 \hat{s}}-\frac{7 \hat{x}^{3}}{12 \hat{s}^{2}}+\frac{\hat{x}^{4}}{8 \hat{s}^{3}} .
\end{aligned}
$$

In accordance with eq. (50) and (51) integration of these expressions gives the value for energy loss of the particle with coordinate $\hat{s}$ in the bunch:

$$
\begin{array}{lll}
\text { region } 1 & \left(2 \hat{s}<\hat{\phi}_{m}\right) \\
& \Delta \hat{\mathcal{E}}_{1} \simeq-\frac{2}{3} \hat{\phi}_{m}^{2}\left(2 \frac{\hat{s}}{\hat{\phi}_{m}}-\frac{7}{5} \frac{\hat{s}^{2}}{\hat{\phi}_{m}^{2}}\right), \\
\text { region } 2 & \left(\hat{\phi}_{m}<2 \hat{s}\right)
\end{array}
$$




$$
\Delta \hat{\mathcal{E}}_{2} \simeq-\frac{2}{3} \hat{\phi}_{m}^{2}\left(1-\frac{1}{4} \frac{\hat{\phi}_{m}}{\hat{s}}+\frac{1}{16} \frac{\hat{\phi}_{m}^{2}}{\hat{s}^{2}}-\frac{1}{80} \frac{\hat{\phi}_{m}^{3}}{\hat{s}^{3}}\right)
$$

Total energy losses of the whole bunch due to the coherent radiation can be calculated as follows:

$$
\begin{array}{ll}
\text { region } 1 \quad & \left(2 \hat{l}_{b}<\hat{\phi}_{m}\right) \\
& \Delta \hat{\mathcal{E}}_{\text {tot }} \simeq \int_{0}^{\hat{l}_{b}} d \hat{s} \Delta \hat{\mathcal{E}}_{1}, \\
\text { region } 2 \quad & \left(\hat{\phi}_{m}<2 \hat{l}_{b}\right) \\
& \Delta \hat{\mathcal{E}}_{t o t} \simeq \int_{0}^{\frac{\dot{\phi}_{m}}{2}} d \hat{s} \Delta \hat{\mathcal{E}}_{1}+\int_{\frac{\dot{\phi}_{m}}{2}}^{l_{b}} d \hat{s} \Delta \hat{\mathcal{E}}_{2} .
\end{array}
$$

Substituting eq. (67) and (68) into eq. (69) and (70), we obtain

$$
\Delta \hat{\mathcal{E}}_{\text {tot }} \simeq-\frac{2}{3} \hat{\phi}_{m}^{2} \hat{l}_{b} \Theta(\hat{p})
$$

where $\hat{p}=2 \hat{l}_{b} / \hat{\phi}_{m}$ and function $\Theta(\hat{p})$ is

$$
\Theta(\hat{p})= \begin{cases}\frac{1}{2} \hat{p}\left(1-\frac{7}{30} \hat{p}\right) & \text { at } \hat{p} \leq 1 \\ 1-\frac{1}{2 \hat{p}}\left[\frac{5}{6}+\ln (\hat{p})\right]-\frac{1}{4 \hat{p}^{2}}+\frac{1}{20 \hat{p}^{3}} & \text { at } \hat{p} \geq 1\end{cases}
$$

The expression for the total energy losses in the bunch written down in dimensional notations has the form:

$$
\Delta \mathcal{E}_{t o t} \simeq \frac{e^{2} N^{2} R}{l_{b}^{2} \gamma^{3}} \Delta \hat{\mathcal{E}}_{t o t}=-\frac{2}{3} \frac{e^{2} N^{2}}{l_{b}} \gamma^{2} \phi_{m}^{2} \Theta\left(\frac{2 l_{b} \gamma^{2}}{R \phi_{m}}\right)
$$

In practically interesting case of $\hat{l}_{b} \gg 1$ the energy loss of a single particle is

$$
\Delta \mathcal{E} \simeq-\frac{2}{3} \frac{e^{2} N}{l_{b}} \gamma^{2} \phi_{m}^{2},
$$


and total energy losses of the bunch are $N$ times larger, i.e.

$$
\Delta \mathcal{E}_{t o t} \simeq-\frac{2}{3} \frac{e^{2} N^{2}}{l_{b}} \gamma^{2} \phi_{m}^{2}
$$

In the latter case the energy losses take place mainly after the magnet within the length of $\sim l_{b} \gamma^{2}$. The radiative interaction force is determined mainly by the renormalized Coulomb fields of the back particles with retarded positions located before the magnet entrance. The characteristic formation length of the radiation before the magnet is also of the order of $l_{b} \gamma^{2}$.

\section{2 "Short" bunch and the magnet is "longer" than the bunch}

In this case $\hat{\phi}_{b} \simeq 2 \hat{l}_{b} \ll 1$ and $\hat{\phi}_{m}>\hat{\phi}_{b}$. To describe this case one can use expressions $F_{a}, F_{b}$ and $F_{d}$ in eq. (66), expression for the particle energy loss (67), etc. The only difference is that now the magnet may not be "short", it may be also "long". When the condition $\hat{\phi}_{m} \gg \hat{\phi}_{b}$ is satisfied, there is steadystate regime (edge effects are not important) and the results (31) and (32) can be used inside the magnet.

\section{3 "Long" magnet, "long" bunch and magnet is "longer" than bunch}

The "long" magnet is the most interesting case from practical point of view. Now we are studying the situation when $\hat{\phi}_{m} \gg 1, \hat{\phi}_{b} \simeq \sqrt[3]{24 \hat{l}_{b}} \gg 1$ and $\hat{\phi}_{b}<\hat{\phi}_{m}$. According to these assumption we can consider only the particles in the bunch for which the condition $\hat{\phi}_{s} \simeq \sqrt[3]{24 \hat{s}} \gg 1$ is satisfied (see eq. (49)). First, we need to simplify the expressions for $F_{a}, F_{b}$ and $F_{d}$ (formulae (50), (56) and (57), respectively). The expressions for $F_{a}$ and $F_{b}$ are simple:

$$
\begin{aligned}
& F_{a} \simeq \begin{cases}0 & \text { at } \hat{\phi}<2^{-2 / 3} \hat{\phi}_{s} \\
-4 / \hat{\phi} & \text { at } 2^{-2 / 3} \hat{\phi}_{s}<\hat{\phi}<\hat{\phi}_{s} .\end{cases} \\
& F_{b} \simeq-\frac{4}{\hat{\phi}_{s}} \quad \text { at } \hat{\phi}_{s}<\hat{\phi}<\hat{\phi}_{m} .
\end{aligned}
$$

The expression for $F_{d}(\hat{s}, \hat{x})$ is complicated when $\hat{x} \sim \hat{\phi}_{s}$, but when $\hat{x} \gg \hat{\phi}_{s}$ it can be written in the simple form:

$$
F_{d} \simeq-\frac{2}{\hat{x}}+\frac{1}{\hat{s}} \quad \text { at } \hat{\phi}_{s} \ll \hat{x} \leq 2 \hat{s}
$$


The energy loss of a particle can be presented as the sum

$$
\Delta \hat{\mathcal{E}}(\hat{s})=\Delta \hat{\mathcal{E}}_{a}+\Delta \hat{\mathcal{E}}_{b}+\Delta \hat{\mathcal{E}}_{d}
$$

where

$$
\begin{aligned}
& \Delta \hat{\mathcal{E}}_{a}=\int_{0}^{\hat{\phi}_{s}} d \hat{\phi} F_{a} \simeq-\frac{8}{3} \ln 2, \\
& \Delta \hat{\mathcal{E}}_{b}=\int_{\hat{\phi}_{s}}^{\dot{\phi}_{m}} d \hat{\phi} F_{b} \simeq-4\left(\frac{\hat{\phi}_{m}}{\hat{\phi}_{s}}-1\right), \\
& \Delta \hat{\mathcal{E}}_{d}=\int_{0}^{2 \hat{s}} d \hat{x} F_{d} \simeq-4 \ln \hat{\phi}_{s}+2+2 \ln 3+\frac{4}{3} \ln 2 .
\end{aligned}
$$

Summing up these expressions, one obtains:

$$
\Delta \hat{\mathcal{E}}(\hat{s}) \simeq-4\left[\frac{\hat{\phi}_{m}}{\hat{\phi}_{s}}+\ln \left(\frac{2^{1 / 3} \hat{\phi}_{s}}{3^{1 / 2}}\right)-\frac{3}{2}\right]
$$

As for the total energy loss of the whole bunch, it can be calculated as follows:

$$
\begin{aligned}
\Delta \hat{\mathcal{E}}_{\text {tot }}= & \int_{0}^{\hat{l}_{b}} d \hat{s} \Delta \hat{\mathcal{E}}(\hat{s}) \simeq \\
& -\frac{1}{4} \hat{\phi}_{m} \hat{\phi}_{b}^{2}\left[1+\frac{2}{9} \frac{\hat{\phi}_{b}}{\hat{\phi}_{m}}\left(\ln \hat{l}_{b}+2 \ln 2-\frac{1}{2} \ln 3-\frac{11}{2}\right)\right] .
\end{aligned}
$$

Remembering that

$$
2 \ln 2-\frac{1}{2} \ln 3-\frac{11}{2} \simeq-4
$$

we can write in dimensional notations the expression for the total energy loss of the bunch:

$$
\Delta \mathcal{E}_{t o t} \simeq-\left(\frac{3^{2 / 3} e^{2} N^{2}}{l_{b}^{4 / 3} R^{2 / 3}}\right)\left(R \phi_{m}\right)\left\{1+\frac{3^{1 / 3} 4}{9} \frac{l_{b}^{1 / 3}}{R^{1 / 3} \phi_{m}}\left[\ln \left(\frac{l_{b} \gamma^{3}}{R}\right)-4\right]\right\}
$$


The applicability region of this formula is given by

$$
\frac{1}{\gamma} \ll\left(\frac{24 l_{b}}{R}\right)^{1 / 3} \leq \phi_{m}
$$

When the second term of eq. (83) in figure brackets can be neglected, one obtains (see (28)):

$$
\Delta \mathcal{E}_{t o t} \simeq-\left(\frac{3^{2 / 3} e^{2} N^{2}}{l_{b}^{4 / 3} R^{2 / 3}}\right)\left(R \phi_{m}\right) .
$$

This result can be obtained in the framework of steady-state approach (see refs. $[8-13,5])$. Therefore, now we have the basis for outlining the applicability region of the results obtained in the papers [8-13,5] with respect to the case of a finite magnet length. With logarithmical accuracy the applicability condition can be written as follows:

$$
\frac{l_{b}^{1 / 3}}{R^{1 / 3} \phi_{m}} \ln \left(\frac{l_{b} \gamma^{3}}{R}\right) \ll 1
$$

In practice this condition may not be satisfied. Indeed, in most cases the value of the logarithm is about $10-20$; the value of $l_{b}^{1 / 3} / R^{1 / 3} \phi_{m}$ is limited with the condition (84) and can be of the order of unity. This means that the practical situations are possible when the condition (84) of applicability of formula (83) is valid and the energy losses are defined with logarithmic term in (83). In other words, the losses in this case take place mainly after the magnet on the length $\sim l_{b} \gamma^{2}$.

\section{4 "Long" magnet, bunch is "much longer" than magnet}

Now let us investigate the case when $1 \ll \hat{\phi}_{m} \ll \hat{\phi}_{b}$. Considering only the particles with positions along the bunch given by $\hat{\phi}_{s} \gg \hat{\phi}_{m}$, we obtain the following features of radiative interaction force acting on these particles. First, the force is negligible inside the magnet. Second, the only term $F_{c}$ is important which describes the interaction of reference particle moving along the straight line after the magnet with the particles with retarded positions before the magnet. This renormalized Coulomb term can be written as follows:

$$
F_{c} \simeq \begin{cases}0 & \text { at } 0<\hat{x}<2 \hat{s} / \hat{\phi}_{m}^{2} \\ -2 / \hat{x}+1 / \hat{s} & \text { at } 2 \hat{s} / \hat{\phi}_{m}^{2}<\hat{x}<2 \hat{s} .\end{cases}
$$


After integration over the trajectory, one obtains the particle energy loss:

$$
\Delta \hat{\mathcal{E}} \simeq-4 \ln \hat{\phi}_{m}+2
$$

In dimensional notations it can be written as

$$
\Delta \mathcal{E} \simeq-\frac{N e^{2}}{l_{b}}\left[4 \ln \left(\gamma \phi_{m}\right)-2\right]
$$

The total energy loss of the bunch is simply $N$ times larger:

$$
\Delta \mathcal{E}_{t o t} \simeq-\frac{N^{2} e^{2}}{l_{b}}\left[4 \ln \left(\gamma \phi_{m}\right)-2\right] .
$$

It should be noted that specific distribution along the trajectory of the energy loss of the particle, when the radiative interaction force (87) is almost absent before some point and then jumps, is the feature of a bunch with stepped profile. Let us discuss the case of smooth density distribution when characteristic bunch length is much more than $R \phi_{m}^{3}$. The radiative interaction force will be smooth but it will decay after some maximum as $1 / x$. The energy loss will be defined by the logarithmic factor as in eq. (88) and will be proportional to the local current density. For example, in the case of Gaussian density distribution (24) the particles energy losses are distributed along the bunch as follows

$$
\Delta \mathcal{E}(s) \simeq-\frac{N e^{2}}{(2 \pi)^{1 / 2} \sigma} \exp \left(-\frac{s^{2}}{2 \sigma^{2}}\right)\left[4 \ln \left(\gamma \phi_{m}\right)-2\right]
$$

and the total energy losses of the whole bunch are

$$
\Delta \mathcal{E}_{t o t} \simeq-\frac{N^{2} e^{2}}{2 \pi^{1 / 2} \sigma}\left[4 \ln \left(\gamma \phi_{m}\right)-2\right]
$$

\section{Discussion}

Let us discuss the results obtained in this paper. First, it is interesting to compare the total energy losses of the whole bunch with the energy of coherent radiation in far zone. The latter one is usually calculated as the integral over frequencies of the spectral density of radiation energy:

$$
\frac{d W_{c o h}}{d \omega}=N^{2} \eta(\omega) \frac{d W}{d \omega}
$$


where $\eta(\omega)$ is the bunch form factor (see, for example, [16]). The form factor for the rectangular bunch of the length $l_{b}$ is:

$$
\eta(\omega)=\left(\sin \frac{\omega l_{b}}{2 c}\right)^{2}\left(\frac{\omega l_{b}}{2 c}\right)^{-2}
$$

The function $d W / d \omega$ in eq. (91) is the spectral density of radiation energy of a single electron. The angular and spectral characteristics of the radiation of an electron moving in an arc of a circle was investigated in ref. [14] ${ }^{1}$. It was shown that in this case the radiation spectrum differs significantly from that of usual synchrotron radiation when electron executes periodical circular motion. In particular, in the latter case the spectral density at low frequencies is proportional to $\omega^{1 / 3}$ (see [18]). Contrary to this, in the case of finite curved track length, the spectral density is constant at $\omega \rightarrow 0$. When the bending angle is small $\left(\phi_{m} \ll 1\right)$ and electron motion is ultrarelativistic, this energy spectral density (see [14]) is the function of the only parameter $\hat{\phi}_{m}=\gamma \phi_{m}$ and can be written:

$$
\frac{d W}{d \omega}=\frac{e^{2}}{\pi c} f_{m}
$$

where

$$
f_{m}=\left(\mu+\frac{1}{\mu}\right) \ln \frac{1+\mu}{1-\mu}-2
$$

and

$$
\mu=\frac{\hat{\phi}_{m} / 2}{\sqrt{1+\left(\hat{\phi}_{m} / 2\right)^{2}}} .
$$

Formula (93) is valid for frequencies $\omega \ll c / L_{s l}$, where $L_{s l}$ is characteristic slippage length in the magnet of the radiation with respect to electron: $L_{s l} \simeq$ $R \phi_{m} / 2 \gamma^{2}+R \phi_{m}^{3} / 24$. Taking into account formula (92) we can estimate that typical frequencies of coherent radiation are below the frequency $\omega \sim c / l_{b}$. It means that we can use the asymptotical expression (93) in the case when $l_{b} \gg L_{s l}$ or, according to our classification, when the bunch is "much longer" than the magnet. Integrating eq. (91) over frequency interval from 0 to infinity, we obtain:

$$
W_{c o h}=\frac{e^{2} N^{2}}{l_{b}} f_{m} .
$$

${ }^{1}$ The same problem was considered later in ref.[17] but the results of the paper [17] are incorrect. 
In the case of "long" magnet $\hat{\phi}_{m} \gg 1$ the function $f_{m}$ reduces to

$$
f_{m}=4 \ln \hat{\phi}_{m}-2
$$

and the coherent radiation energy exactly coincides with the bunch energy losses given by eq. (90) taken with opposite sign. In the opposite limit of "short" magnet, $\hat{\phi}_{m} \ll 1$, we obtain:

$$
f_{m}=\frac{2}{3} \hat{\phi}_{m}^{2} \text {. }
$$

There is complete agreement with formula (73).

The case when the bunch is "much longer" than the magnet is interesting from methodological point of view. Indeed, in the previous section we have found that the bunch loses energy under the action of radiative interaction force mainly after the magnet on the length of $\sim l_{b} \gamma^{2}$. On the other hand, an observer in far zone detects the radiation pulse and, calculating the retarded positions of radiators, he can conclude that electrons of the bunch radiated when they were inside the magnet. The methodological questions of such kind (electrons radiate when the radiative force does not work on them and vice versa) are related not only to coherent radiation processes. They arise also in single particle electrodynamics and were discussed by different authors (see, for example, ref.[19]).

We should also discuss the applicability region of the theory presented in this paper with respect to the importance of the length of $\sim l_{b} \gamma^{2}$ before and after the magnet giving the contribution into the CSR effects. These linear sections are important when the condition (86) is violated (we mean here the practically important case of a "long' magnet). In this case the results of this paper are valid when particles are not disturbed with any external fields on the parts of the trajectory of $\sim l_{b} \gamma^{2}$ before and after the magnet. In practice this condition may not be satisfied $\left(l_{b} \gamma^{2}\right.$ can be of the order of tens of meters or more) and one has to use the results of this paper carefully.

In this paper we studied in detail the case of the electron bunch with rectangular density profile, because we were interested mainly in the physical aspects of the problem. Now let us show how the obtained results can be extended to the case of the bunch with an arbitrary density distribution. First, formulae obtained in section 4 for the radiative interaction of two electrons can be integrated directly (at least, numerically) with any density distribution. Second, the analytical results obtained in the sections 5 and 6 for the rectangular density distribution can simplify significantly the solution of the problem with an arbitrary density distribution. Let us illustrate this idea with the calculation of the transition process when the bunch enters the magnet. Let the bunch 
have the density distribution $\lambda(s)$ which satisfies the condition (17). One can consider this bunch as a composition of rectangular bunches, i.e. to calculate the radiative interaction force at some point $s$ one can add together the forces from partial bunches with a length $\left(s-s^{\prime}\right)$ and a linear density $d s^{\prime}\left[d \lambda\left(s^{\prime}\right) / d s^{\prime}\right]$. One should remember that always $s^{\prime}<s$. To calculate the contribution of the partial bunches one can use the expressions (74) and (75). As a result, the rate of the energy change for a particle in the bunch can be written as follows:

$$
\begin{aligned}
\frac{d \mathcal{E}(s, \phi)}{d(c t)}= & -\frac{2 e^{2}}{3^{1 / 3} R^{2 / 3}}\left\{\left(\frac{24}{R \phi^{3}}\right)^{1 / 3}\left[\lambda\left(s-\frac{R \phi^{3}}{24}\right)-\lambda\left(s-\frac{R \phi^{3}}{6}\right)\right]\right. \\
& \left.+\int_{s-R \phi^{3} / 24}^{s} \frac{d s^{\prime}}{\left(s-s^{\prime}\right)^{1 / 3}} \frac{d \lambda\left(s^{\prime}\right)}{d s^{\prime}}\right\} .
\end{aligned}
$$

This formula allows one to calculate the distribution of the rate of the energy change of the particles as a function of a bending angle $\phi$ when the bunch enters the magnet. Let us consider the Gaussian density distribution (24). In this case the expression (95) can be written in the following form:

$$
\frac{d \mathcal{E}}{d(c t)}=-\frac{2 e^{2} N}{3^{1 / 3}(2 \pi)^{1 / 2} R^{2 / 3} \sigma^{4 / 3}} G(\xi, \rho)
$$

where the function $G(\xi, \rho)$ is given with expression

$$
G(\xi, \rho)=\rho^{-1 / 3}\left[e^{-(\xi-\rho)^{2} / 2}-e^{-(\xi-4 \rho)^{2} / 2}\right]+\int_{\xi-\rho}^{\xi} \frac{d \xi^{\prime}}{\left(\xi-\xi^{\prime}\right)^{1 / 3}} \frac{d}{d \xi^{\prime}} e^{-\left(\xi^{\prime}\right)^{2} / 2}
$$

Here $\xi=s / \sigma$ and $\rho=R \phi^{3} / 24 \sigma$. When $\rho \ll 1$, the function $G(\xi, \rho)$ reduces to

$$
G(\xi, \rho) \simeq-\frac{9}{2} \xi \exp \left(-\xi^{2} / 2\right) \rho^{2 / 3}
$$

In opposite case, when $\rho \rightarrow \infty$, the expression (97) tends to the steady-state solution (25). In Fig.6 the function (96) is plotted and compared with the results of numerical simulations of ref. [6]. One can see that analytical and numerical results are in a good agreement. 


\section{Acknowledgement}

We wish to thank R. Brinkmann, Ya.S. Derbenev, M. Dohlus, K. Floettmann, T. Limberg, H. Mais, A. Piwinski, J. Rossbach, V.D. Shiltsev and D. Trines for many useful discussions. 


\section{References}

[1] Status report on TESLA,NLC,JLC are given in : Proceedings of the LC'92 ECFA Workshop on $e^{+} e^{-}$Linear Colliders (July, 25-August, 2, 1993, Germany), MPI-PhE/93-14, ECFA 93-154.

[2] R. Tatchyn et al., Nucl. Instr. and Methods A375(1996)274.

[3] R. Brinkmann, G Materlik, J. Rossbach, J.R. Schneider, B.-H. Wiik, "An X-ray FEL laboratory as part of linear collider design", presented at XVIII International Free Electron Laser Conference (Rome,1996); DESY print, TESLA-FEL 96-13 (1996).

[4] J. Rossbach, Nucl. Instr. and Methods A375(1996)269.

[5] Ya.S. Derbenev, J. Rossbach, E.L. Saldin, V.D. Shiltsev, DESY Print, TESLAFEL 95-05, Hamburg(1995).

[6] M. Dohlus, T. Limberg, "Emittance Growth due to Wake Fields on Curved Bunch Trajectories", presented at XVIII International Free Electron Laser Conference (Rome,1996); DESY print, TESLA-FEL 96-13 (1996).

[7] H. Edwards, editor, TESLA Test Facility Linac: Conceptual Design Report, Hamburg, DESY, December 5, (1994).

[8] L.I. Schiff, Rev. Sci. Instr. 17(1946)6.

[9] J. Schwinger, "On radiation by electrons in a betatron", 1945 (unpublished), see references in [10].

[10] J.S. Nodvick and D.S. Saxon, Phys. Rev. 96(1954)180.

[11] I.E. Tamm, Report of the Institute of Physics, Acad. of Sciences, USSR, 1948 (in Russian).

[12] L.V. Iogansen and M.S. Rabinovich, Sov. Phys. JETP, vol.37(10), 1960, p.83.

[13] J.B. Murphy, S. Krinsky and R.L. Glukstern, Proc. of IEEE PAC 1995, Dallas(1995).

[14] V.G. Bagrov, I.M. Ternov and N.I. Fedosov, Sov. Phys. JETP, vol.55(5), 1982, p.835; Phys. Rev. D28 (1983)2464.

[15] J.D. Jackson, Classical Electrodynamics, (Wiley, New York, 1975).

[16] H. Wiedemann, Particle Accelerator Physics II, (Springer-Verlag, BerlinHeidelberg, 1995).

[17] D.J. Wingham, Phys. Rev. D35(1987)2584.

[18] J. Schwinger, Phys. Rev. 75(1949)1912.

[19] V.L. Ginzburg, Applications of Electrodynamics in Theoretical Physics and Astrophysics, (Gordon and Breach Science Publishers, New York, 1989). 


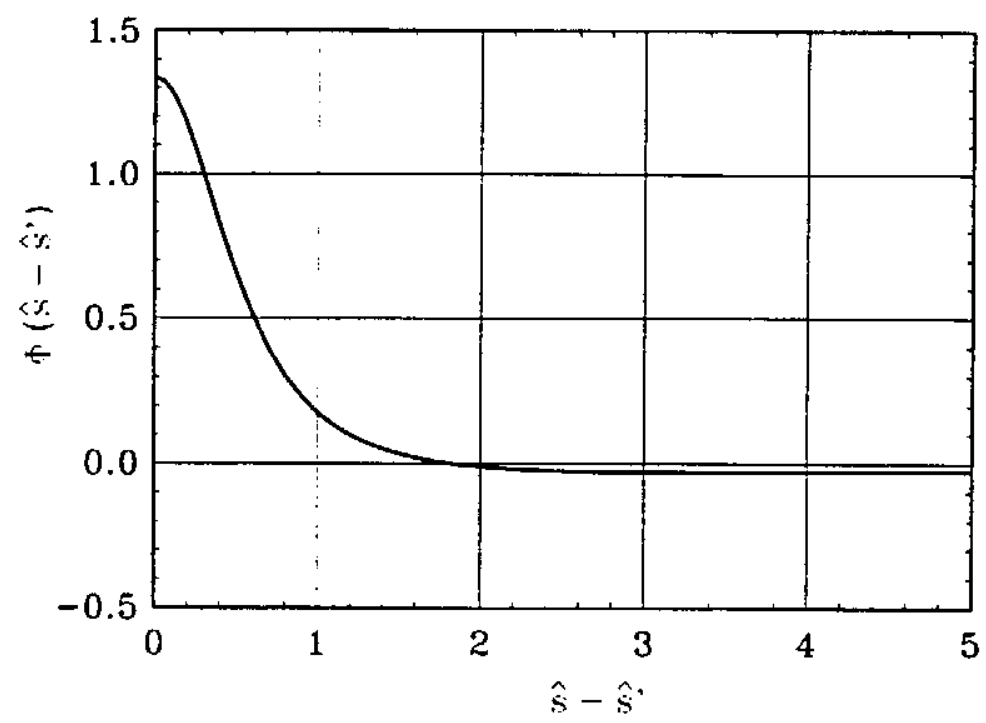

Fig. 1. The radiative interaction function (13) between two electrons travelling in a circle.

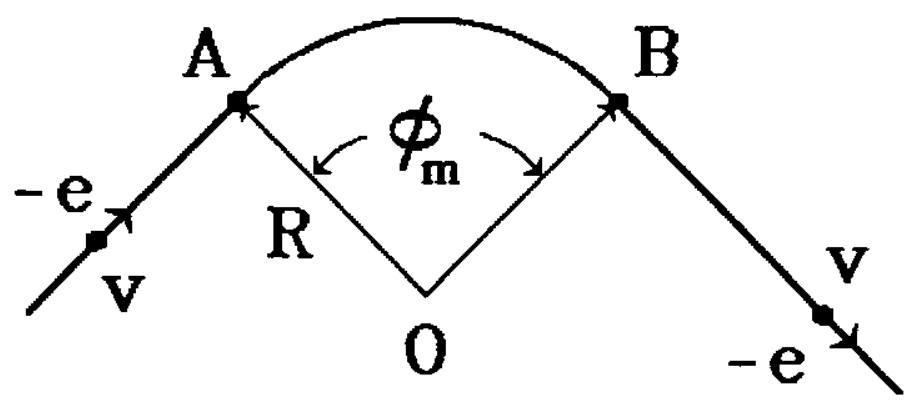

Fig. 2. Trajectory of a particle passing a bending magnet. 

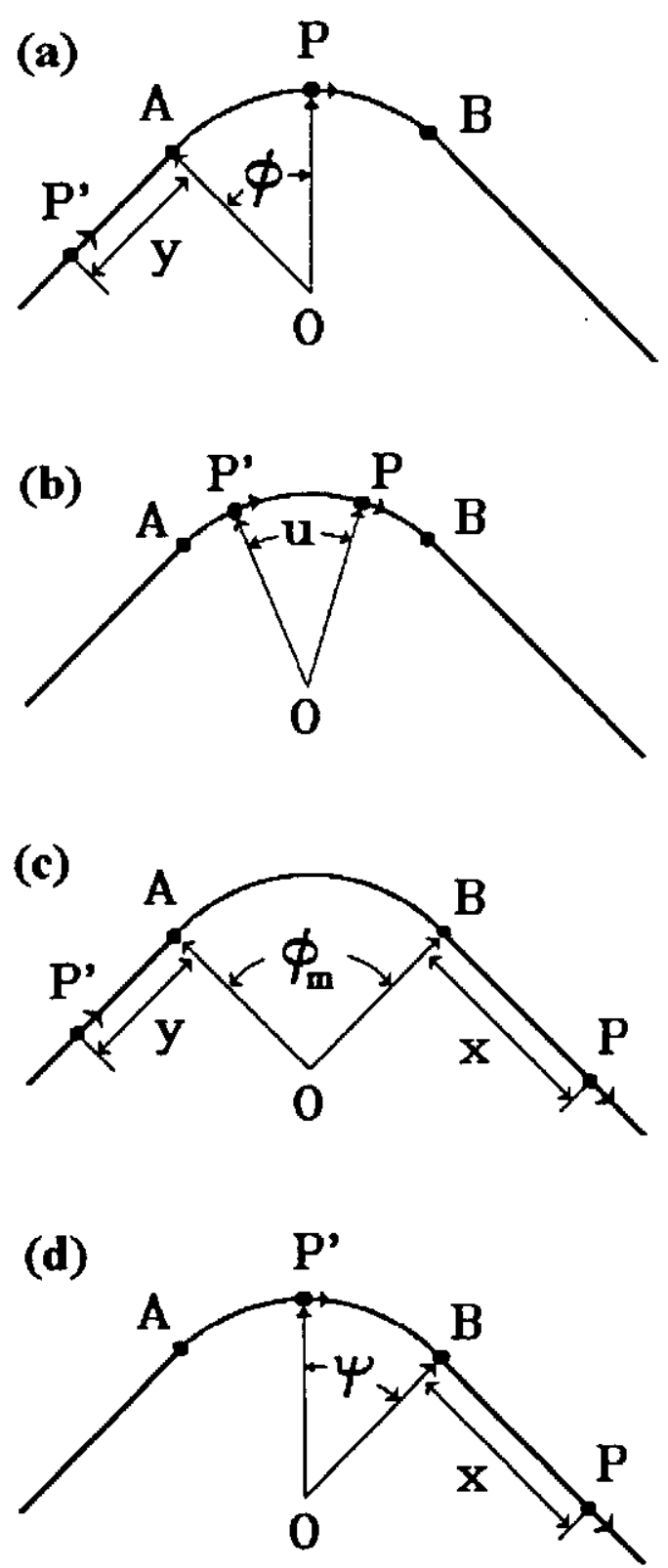

Fig. 3. Relative configuration of source point $P^{\prime}$ and field point $P$ for a system of two electrons passing a bending magnet. 


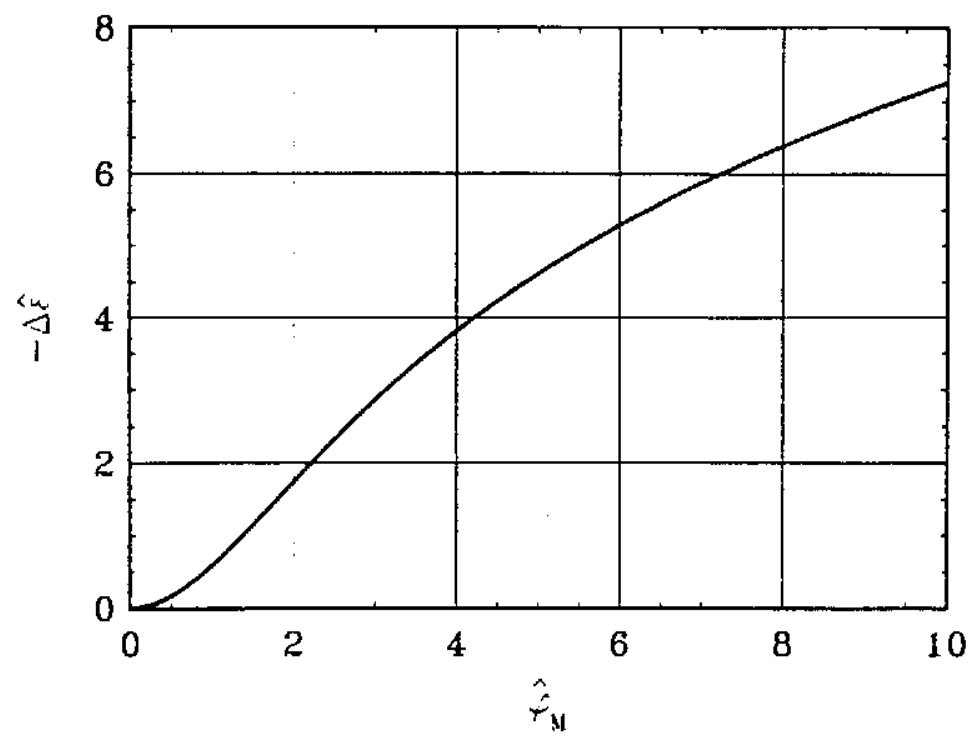

Fig. 4. The normalized energy change of a particle versus the normalized bending angle of a magnet. The particle position along the rectangular bunch is defined with the condition $\hat{\phi}_{s} \gg \hat{\phi}_{m}$.

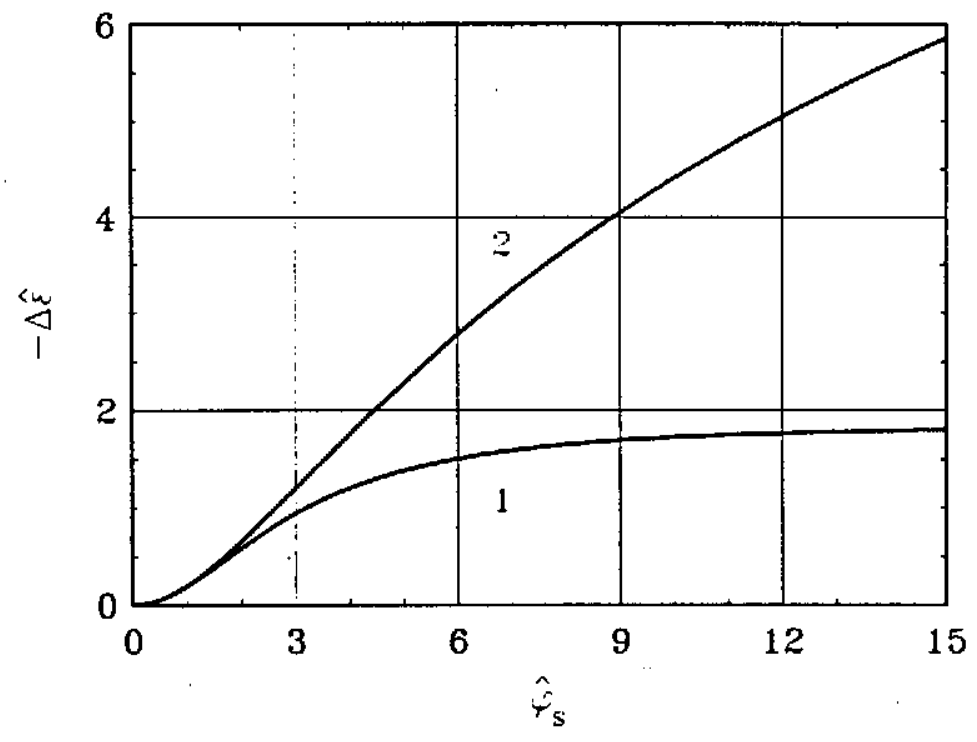

Fig. 5. The normalized energy change of a particle versus its reduced position $\hat{\phi}_{s}$ along the rectangular bunch when $\hat{\phi}_{1}<\hat{\phi}_{m}$. The curve (1) corresponds to the transition region when bunch enters the magnet (see the first integral in eq. (50)) and the curve (2) shows the energy change after the magnet (see the third integral in eq. (50)). 
(a)

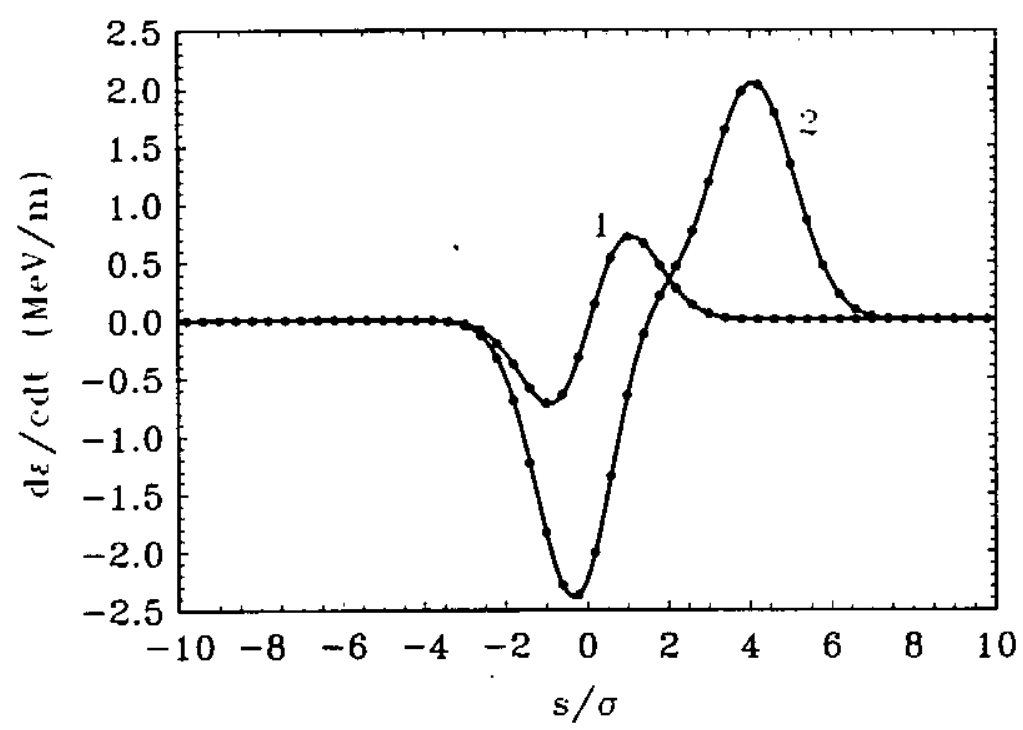

(b)

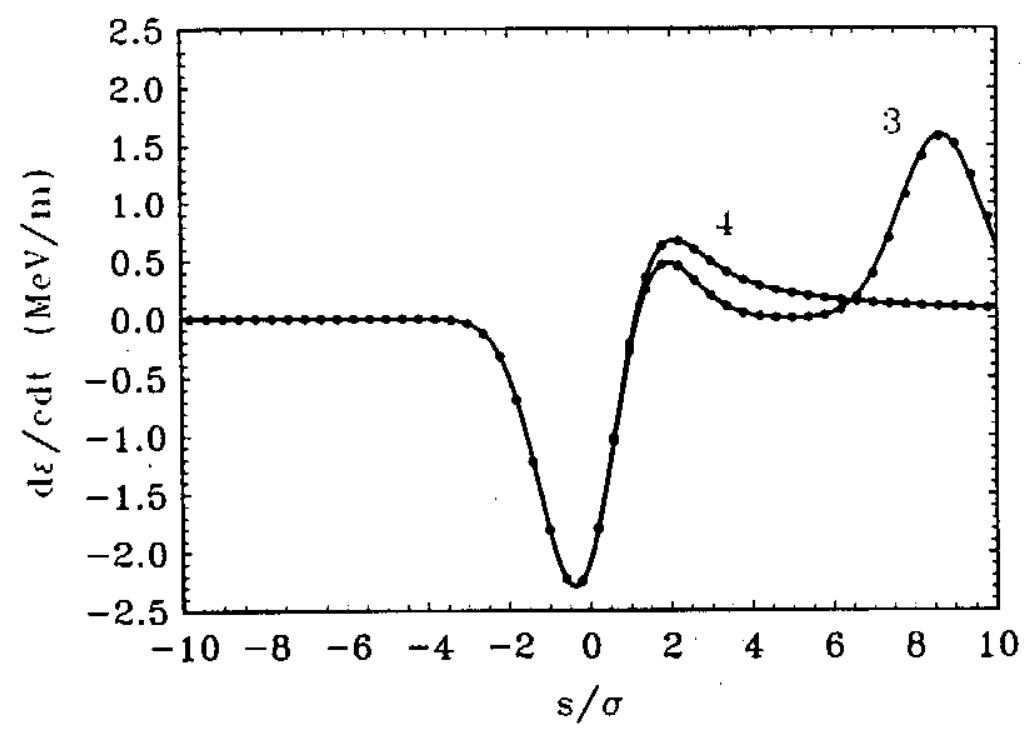

Fig. 6. The rate of an electron energy change as a function of its position along the Gaussian bunch entering the magnet. Curve (1) in the graph (a): $5 \mathrm{~cm}$ after the begin of the magnet. Curve (2) in the graph (a): $14 \mathrm{~cm}$ after the begin of the magnet. Curve (3) in the graph (b): $18 \mathrm{~cm}$ after the begin of the magnet. Curve (4) in the graph (b): steady state. The curves are the results of calculations with formula (96) and the circles are the results of numerical simulations presented in ref. [6]. The parameters are as follows: $R=1.5 \mathrm{~m}, \sigma=50 \mu \mathrm{m}, q=1 \mathrm{nC}$. 